\title{
A!
}

This is an electronic reprint of the original article.

This reprint may differ from the original in pagination and typographic detail.

Brange, Fredrik; Menczel, Paul; Flindt, Christian

\section{Photon counting statistics of a microwave cavity}

Published in:

Physical Review B

DOI:

10.1103/PhysRevB.99.085418

Published: 14/02/2019

Document Version

Publisher's PDF, also known as Version of record

Please cite the original version:

Brange, F., Menczel, P., \& Flindt, C. (2019). Photon counting statistics of a microwave cavity. Physical Review $B, 99(8), 1-14$. [085418]. https://doi.org/10.1103/PhysRevB.99.085418

This material is protected by copyright and other intellectual property rights, and duplication or sale of all or part of any of the repository collections is not permitted, except that material may be duplicated by you for your research use or educational purposes in electronic or print form. You must obtain permission for any other use. Electronic or print copies may not be offered, whether for sale or otherwise to anyone who is not an authorised user. 


\title{
Photon counting statistics of a microwave cavity
}

\author{
Fredrik Brange, ${ }^{1}$ Paul Menczel, ${ }^{2}$ and Christian Flindt ${ }^{2}$ \\ ${ }^{1}$ Department of Physics and NanoLund, Lund University, Box 188, SE-221 00 Lund, Sweden \\ ${ }^{2}$ Department of Applied Physics, Aalto University, 00076 Aalto, Finland
}

(Received 30 August 2018; published 14 February 2019)

\begin{abstract}
The development of microwave photon detectors is paving the way for a wide range of quantum technologies and fundamental discoveries involving single photons. Here, we investigate the photon emission from a microwave cavity and find that distribution of photon waiting times contains information about few-photon processes, which cannot easily be extracted from standard correlation measurements. The factorial cumulants of the photon counting statistics are positive at all times, which may be intimately linked with the bosonic quantum nature of the photons. We obtain a simple expression for the rare fluctuations of the photon current, which is helpful in understanding earlier results on heat-transport statistics and measurements of work distributions. Under nonequilibrium conditions, where a small temperature gradient drives a heat current through the cavity, we formulate a fluctuation-dissipation relation for the heat noise spectra. Our work suggests a number of experiments for the near future and it offers theoretical questions for further investigation.
\end{abstract}

DOI: 10.1103/PhysRevB.99.085418

\section{INTRODUCTION}

The development of quantum technologies relies on the ability to control, transmit, and detect single quanta of light, heat, and charge [1]. Much effort has thus been devoted to the manipulation of individual photons [2,3], phonons [4,5], and electrons [6] at the nanoscale. Electrons [7-10] and photons [11] can be emitted on demand and in some cases detected with single-particle resolution. In one approach, single electrons are captured in a quantum dot, whose charge state is read out using a capacitively coupled conductor [12]. Photons, by contrast, are uncharged with energies in nanoscale systems that can be very small (in the microwave range), requiring highly sensitive detectors [13].

Recently, it has been suggested that microwave photons may be detected in a calorimetric approach [14-16]. A resistive environment is monitored in real-time using ultrasensitive thermometry with dips and peaks in the temperature corresponding to the emission or absorption of single photons. In another proposal, microwave photons are detected using Josephson junctions $[17,18]$. Very recently, a quantum nondemolition detector for propagating microwave photons was realized [19]. Such single-photon detectors are paving the way for a wide range of applications within quantum thermodynamics [20], feedback control [21], and quantum information processing [22]. Moreover, they may help address fundamental questions regarding heat transport, entropy production, and fluctuation relations at the nanoscale [23].

In this paper, we investigate the photon counting statistics of a microwave cavity at the single-particle level [24-27], see Fig. 1. The problem is simple to formulate, yet, surprisingly rich in physics. By combining a generating function technique with the method of characteristics, we obtain a full analytic solution for the photon counting statistics on all relevant timescales. The short-time physics can be characterized by the distribution of photon waiting times [28-33], which contains information about few-photon processes which cannot easily
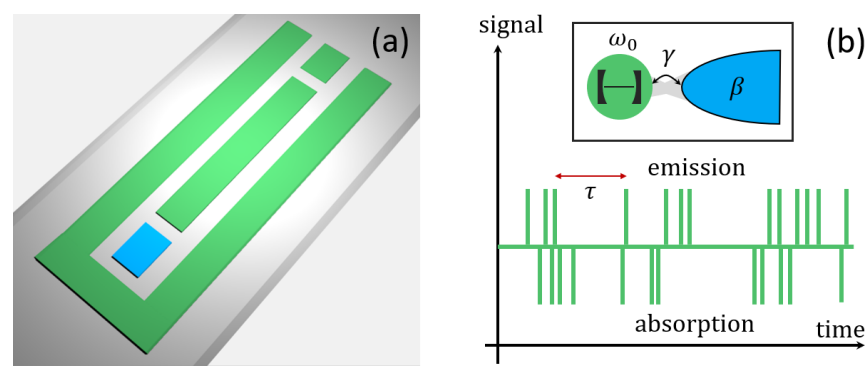

FIG. 1. Photon emission from a microwave cavity. (a) Photons are transmitted between a microwave cavity (in green) and an external heat bath (in blue). (b) A possible time trace of emission and absorption events measured by a single-photon detector. The waiting time between photon emissions is denoted by $\tau$. The setup is shown schematically in the inset. The cavity with frequency $\omega_{0}$ is coupled at the rate $\gamma$ to an external heat bath at the inverse temperature $\beta$.

be extracted from standard correlation measurements. The factorial cumulants of the counting statistics [34-40] are positive at all times, and we conjecture that this behavior is linked with the bosonic quantum nature of the photons. At long times, we find a simple expression for the rare fluctuations of the photon current which may explain earlier results on heat transport statistics [41] and measurements of work distributions [42]. Finally, we consider a nonequilibrium situation, where a temperature gradient drives a heat current through the cavity. Here, we obtain fluctuation-dissipation theorems in the linear and weakly nonlinear regimes, and we formulate a relation between the heat noise spectra and the response of the system to small perturbations of the cavity frequency.

\section{MICROWAVE CAVITY}

We consider the photon emission from a microwave cavity with the Hamiltonian $\hat{H}=\hbar \omega_{0}\left(\hat{a}^{\dagger} \hat{a}+\frac{1}{2}\right)$, where $\hat{a}^{\dagger}(\hat{a})$ 
creates (annihilates) photons with frequency $\omega_{0}$. The density matrix of the cavity $\hat{\rho}(t)$ evolves according to the Lindblad equation [43],

$$
\frac{d \hat{\rho}}{d t}=\mathcal{L} \hat{\rho}=-\frac{i}{\hbar}[\hat{H}, \hat{\rho}]+\gamma\left([\bar{n}+1] \mathcal{D}[\hat{a}] \hat{\rho}+\bar{n} \mathcal{D}\left[\hat{a}^{\dagger}\right] \hat{\rho}\right),
$$

where $\bar{n}=1 /\left(e^{\beta \hbar \omega_{0}}-1\right)$ is the average occupation of the cavity in equilibrium at the inverse temperature $\beta=1 /\left(k_{B} T\right)$, and $\gamma$ governs the photon emission and absorption rates. The Liouvillian $\mathcal{L}$ captures both the unitary evolution described by $\hat{H}$ and the incoherent dynamics given by the dissipators, $\mathcal{D}[\hat{a}] \hat{\rho} \equiv \hat{a} \hat{\rho} \hat{a}^{\dagger}-\frac{1}{2}\left\{\hat{a}^{\dagger} \hat{a}, \hat{\rho}\right\}$. To be specific, we formulate our problem in terms of a microwave cavity [24-27]; however, our findings below are clearly valid for any other bosonic degree of freedom that can be treated as a dissipative quantum harmonic oscillator, for instance, a nanomechanical resonator [5]. Moreover, the heat bath can be either bosonic or fermionic (see Appendix A), as, for example, an electronic reservoir, where the emission and absorption of single photons give rise to dips and peaks in the temperature, which can be measured using ultrasensitive thermometry [14-16].

\section{PHOTON COUNTING STATISTICS}

To investigate the photon counting statistics, we unravel the Lindblad equation with respect to the number of photons $m$ emitted during the time span $[0, t][44]$. Hence, we resolve the density matrix as $\hat{\rho}(t)=\sum_{m} \hat{\rho}(m, t)$, from which we obtain the photon counting statistics, $P(m, t)=\operatorname{Tr}\{\hat{\rho}(m, t)\}$. The density matrices evolve as $\frac{d}{d t} \hat{\rho}(m, t)=\left(\mathcal{L}-\mathcal{J}_{e}\right) \hat{\rho}(m, t)+$ $\mathcal{J}_{e} \hat{\rho}(m-1, t)$, where $\mathcal{J}_{e} \hat{\rho}=\gamma(\bar{n}+1) \hat{a} \hat{\rho} \hat{a}^{\dagger}$ is the superoperator for the photon emission current. The equations of motion do not couple populations of the density matrices to the coherences; however, the populations are mutually coupled. To decouple the system of equations, we introduce the generating function $\mathcal{G}(s, q, t) \equiv \sum_{n, m}\langle n|\hat{\rho}(m, t)| n\rangle e^{m s+n q}$, where $s$ and $q$ are conjugate variables to the number of emitted photons $m$ and the cavity occupation number $n$, respectively. The generating function obeys the partial differential equation (see Appendix A),

$$
\partial_{t} \mathcal{G}(s, q, t)=[f(s, q)+g(q)] \partial_{q} \mathcal{G}(s, q, t)+g(q) \mathcal{G}(s, q, t),
$$

with $f(s, q)=\gamma(\bar{n}+1)\left(e^{s-q}-1\right)$ and $g(q)=\gamma \bar{n}\left(e^{q}-1\right)$. Remarkably, the differential equation can be solved analytically using the method of characteristics [45]. The generating function contains statistical information both about the number of photons in the cavity [46-49] and the number of photons that have been emitted [50-52]. Here, we focus on the photon emission statistics with the moment-generating function $(\mathrm{MGF}) \mathcal{M}(s, t) \equiv \sum_{m} P(m, t) e^{m s}=\mathcal{G}(s, 0, t)$. In thermal equilibrium, we find (see Appendix B)

$$
\mathcal{M}(s, t)=\frac{2 \xi e^{\gamma t / 2}}{2 \xi \cosh \left[\frac{\xi \gamma t}{2}\right]+\left(1+\xi^{2}\right) \sinh \left[\frac{\xi \gamma t}{2}\right]},
$$

with $\xi=\sqrt{1-4 \bar{n}(1+\bar{n})\left(e^{s}-1\right)}$. This expression holds on all timescales, where Eq. (1) is valid [53], and it is important for our further analysis of the photon emission statistics. With $\gamma$ fixing the timescale, we are left with a single dimensionless parameter, namely the mean occupation number $\bar{n}$, controlled by the temperature $T$.

\section{WAITING TIME DISTRIBUTION}

We first analyze the waiting time $\tau$ between photon emissions [28-30]. Recently, waiting time distributions (WTDs) have been measured both for photon emission [3] and electron tunneling [54]. The WTD can be obtained as $\mathcal{W}(\tau)=$ $\langle\tau\rangle \partial_{\tau}^{2} \Pi(\tau)$, where $\langle\tau\rangle$ is the mean waiting time and $\Pi(\tau)$ is the probability that no photons are emitted in a time span of duration $\tau$ [31,32]. Physically, the time derivatives correspond to a photon emission at the beginning and the end of the time interval [33]. From the definition of the MGF, we have $\Pi(\tau)=\mathcal{M}(-\infty, \tau)$ and then obtain (see Appendix C)

$$
\mathcal{W}(\tau)=\Gamma \gamma \bar{\gamma} \frac{\gamma+6 \Gamma+(\gamma+2 \Gamma) \cosh [\bar{\gamma} t]+\bar{\gamma} \sinh [\bar{\gamma} t]}{\left(\bar{\gamma} \cosh \left[\frac{\bar{\gamma} t}{2}\right]+(\gamma+2 \Gamma) \sinh \left[\frac{\bar{\gamma} t}{2}\right]\right)^{3}} e^{\frac{\gamma t}{2}} .
$$

Here, we have used that the average emission rate is $\left\langle J_{e}\right\rangle=$ $\gamma \bar{n}(1+\bar{n}) \equiv \Gamma$, and we have defined $\bar{\gamma} \equiv \gamma(1+2 \bar{n})$.

Figure 2(a) shows WTDs for different temperatures. The distributions start off at a finite value, $\mathcal{W}(0)=2 \Gamma$, and then decay monotonically to zero at long times. This behavior should be contrasted with that of noninteracting fermions, for which the distributions are typically suppressed
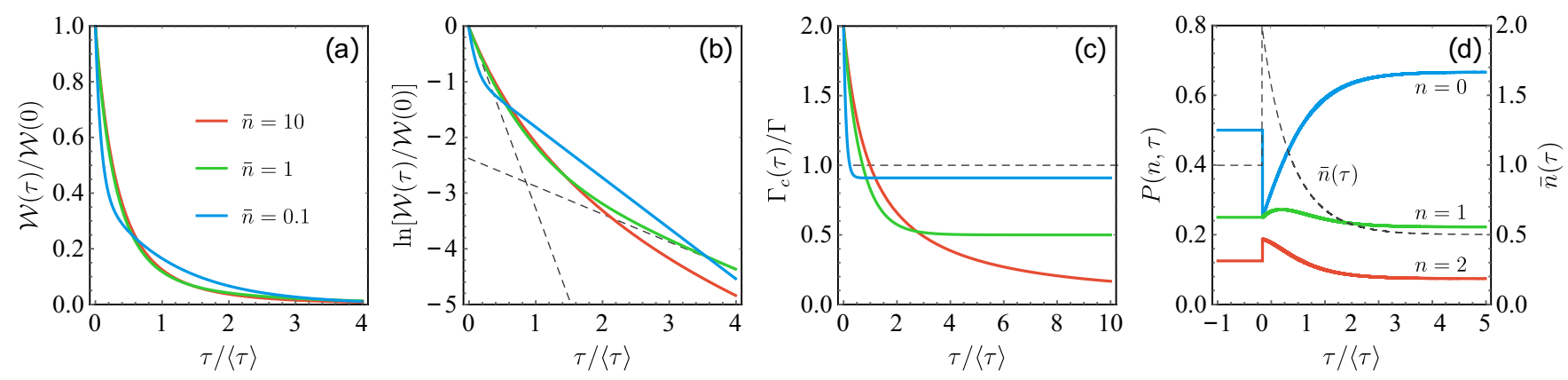

FIG. 2. Photon waiting times. (a) Distribution of waiting times for different average occupations of the cavity, $\bar{n}=0.1,1,10$. (b) The fast and slow decay rates are clearly visible on a logarithmic scale with the dashed lines corresponding to $\bar{n}=1$. (c) Conditional emission rate given that the last emission occurred at the time $\tau=0$. The dashed line corresponds to a Poisson process. (d) Probability of having $n$ photons (left axis) and mean number of photons (right axis) in the cavity given that the last emission occurred at the time $\tau=0$. For $\tau<0$, the cavity is populated according to a Boltzmann distribution with $\bar{n}=1$. 
at short times due to the Pauli principle [31,32]. Similar to the recent experiments [3,54], the WTDs are double exponential. For short times, $\Gamma \tau \ll 1$, we have $\mathcal{W}(\tau) \simeq$ $2 \Gamma \exp (-\gamma[6 \bar{n}(\bar{n}+1)+1] \tau / 2)$, showing that the fast decay rate increases quadratically with the mean occupation $\bar{n}$, and not just linearly as one might expect. At long times, $\Gamma \tau \gg 1$, we have $\mathcal{W}(\tau) \simeq \frac{4 \Gamma \gamma \bar{\gamma}}{(\gamma+\bar{\gamma}+2 \Gamma)^{2}} \exp (-\gamma \bar{n} \tau)$ with the slow decay rate given by $\gamma \bar{n}$. Figure 2(b) illustrates the crossover between these limiting behaviors.

The increased decay rate at short times is a signature of photon bunching. This phenomenon is illustrated in Fig. 2(c), showing the conditional emission rate, $\Gamma_{c}(\tau)=$ $\mathcal{W}(\tau) / \int_{\tau}^{\infty} \mathcal{W}(u) d u$, at the time $\tau$ after the last photon emission. Due to the photon bunching, the rate is enhanced at short times and suppressed at long times. The bunching also affects the number of photons in the cavity at the time $\tau$ after the last photon emission, see Fig. 2(d). An application of Bayes' theorem shows that the expected number of photons in the cavity increases by a factor of two directly after an emission event (see Appendix C). At longer times, with no subsequent emissions, it is increasingly likely that the cavity is empty, and it eventually reaches a Boltzmann distribution, albeit with an average photon number $\bar{n} /(1+\bar{n})$ suppressed below one.

\section{CORRELATION FUNCTION}

A different perspective on the short-time physics is provided by the $g^{(2)}$-function $[2,3,5,55]$. The $g^{(2)}$-function is proportional to the probability that a photon is emitted at the time $\tau$, given that a photon was emitted at the time $\tau=0$. Unlike the WTD, other photon emissions may have occurred during this time span. The correlation function can be obtained from Eq. (3), and we find (see Appendix D)

$$
g^{(2)}(\tau)=1+e^{-\gamma|\tau|} .
$$

This is the $g^{(2)}$-function for chaotic thermal light as well as for other noninteracting bosons, for example, thermal phonons as shown in recent experiments [5]. Equations (4) and (5) are important for the recurring discussion about possible connections between the WTD and the $g^{(2)}$-function. For renewal processes, where consecutive waiting times are uncorrelated, the two functions are related in Laplace space as $g^{(2)}(s)\left\langle J_{e}\right\rangle=$ $\mathcal{W}(s) /[1-\mathcal{W}(s)][32,33,56,56]$. This relation does not hold for our cavity, since it does not return to the same state after each emission. Moreover, unlike the $g^{(2)}$-function, the WTD depends on temperature, showing that the two are not equivalent.

\section{FACTORIAL CUMULANTS}

To investigate the transition from short to long observation times, we consider the factorial cumulants of the photon counting statistics [34,34-40]. The factorial cumulants are defined as $\left\langle\left\langle m^{k}\right\rangle\right\rangle_{F}=\langle\langle m(m-1) \ldots(m-k+1)\rangle\rangle$, where $\left\langle\left\langle m^{k}\right\rangle\right\rangle=\left.\partial_{s}^{k} \ln \mathcal{M}(s, t)\right|_{s=0}$ are the ordinary cumulants of order $k$. The counting statistics of noninteracting electrons in a two-terminal setup is always generalized binomial [57-59], and the sign of the factorial cumulants alternates with the order $k$ [36-39]. By contrast, for the photon cavity we find

$$
\begin{aligned}
\langle\mid m\rangle\rangle_{F} & =\gamma t \bar{n}(1+\bar{n}), \\
\left\langle\left\langle m^{2}\right\rangle\right\rangle_{F} & =2 \gamma t\left[1+\frac{e^{-\gamma t}-1}{\gamma t}\right] \bar{n}^{2}(1+\bar{n})^{2}, \\
\left\langle\left\langle m^{3}\right\rangle\right\rangle_{F} & =12 \gamma t\left[1+e^{-\gamma t}+2 \frac{e^{-\gamma t}-1}{\gamma t}\right] \bar{n}^{3}(1+\bar{n})^{3},
\end{aligned}
$$

with similar expressions for the higher factorial cumulants, which are positive. These results suggest that the quantum statistics of the particles, being bosons or fermions, is intimately linked with the sign of the factorial cumulants, consistently with earlier works on photon counting statistics $[34,35]$. At long observation times, we have $\left\langle\left\langle m^{k}\right\rangle_{F} \propto \gamma t \bar{n}^{k}(1+\bar{n})^{k}\right.$, showing that the photon counting statistics is nearly Poissonian at low temperatures, where only the first factorial cumulant is nonzero.

\section{LONG-TIME STATISTICS}

To complete the discussion of the long-time limit, we analyze the large-deviation statistics of the photon emission current [60]. To this end, we evaluate the counting statistics $P\left(J_{e}, t\right)=\frac{1}{2 \pi i} \int_{-i \pi}^{i \pi} d s e^{t\left[\Theta(s)-s J_{e}\right]}$ in the long-time limit, where $\Theta(s)=\lim _{t \rightarrow \infty} \ln [\mathcal{M}(s, t)] / t$ is the cumulant generating function for the photon emission current $J_{e}=m / t$,

$$
\Theta(s)=\frac{\gamma}{2}\left(1-\sqrt{1-4\left(e^{s}-1\right) \bar{n}(1+\bar{n})}\right) .
$$

The large-deviation statistics of the emission current can be evaluated in a saddle-point approximation,

$$
\frac{\ln \left[P\left(J_{e}, t\right)\right]}{t} \simeq \Theta\left(s_{\mathrm{o}}\right)-s_{\mathrm{o}} J_{e},
$$

where $s_{0}=s_{0}\left(J_{e}\right)$ solves the saddle-point equation $\Theta^{\prime}\left(s_{0}\right)=$ $J_{e}$. Figure 3(a) shows the large-deviation statistics for different temperatures. With increasing temperature, the distributions become strongly non-Poissonian and large emission currents are more likely. For large currents, the saddle-point $s_{\mathrm{o}}$ must be close to the square-root singularity of $\Theta(s)$ at $s=s_{c}$, where $\Theta\left(s_{c}\right)=\gamma / 2$ and the derivative $\Theta^{\prime}\left(s_{c}\right)$ diverges. With $s_{\mathrm{o}} \simeq$ $s_{c}=2 \ln \left[\cosh \left(\beta \hbar \omega_{0} / 2\right)\right] \simeq \beta \hbar \omega_{0}$ for $\beta \hbar \omega_{0} \gg 1$, the largedeviation statistics becomes (see Appendix E)

$$
\frac{\ln \left[P\left(J_{e}, t\right)\right]}{t} \simeq \gamma / 2-\beta \hbar \omega_{0} J_{e}, J_{e} \gg \gamma .
$$

This expression agrees well with the exact results in Fig. 3(a). As we discuss below, it provides an analytic understanding of the linear dependence on the heat current and the inverse temperature observed in numerical calculations of the largedeviation statistics in phononic heat transport [41]. A similar reasoning might also be helpful in understanding the tails of the work distributions measured for a microcantilever [42].

\section{HEAT TRANSPORT}

Our analysis can be extended to setups with the cavity coupled to several reservoirs kept at different temperatures, thus providing an interesting opportunity to investigate the 

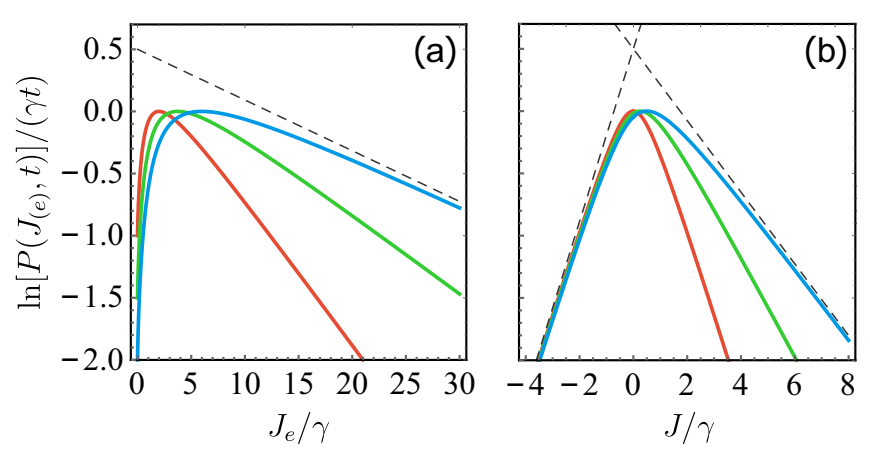

FIG. 3. Large-deviation statistics of the photon current. (a) Analytic results for the distribution of the photon emission current $J_{e}$ from a cavity coupled to a single reservoir with $\bar{n}=1$ (red), 1.5 (green), and 2 (blue). The dashed line is based on the branch-point of the cumulant generating function in Eq. (7), and it is given by Eq. (9) at low temperatures. (b) Distribution of the current $J$ running via the cavity between a hot and a cold reservoir with $\bar{n}_{c}=1, \bar{n}_{h}=1$ (red), $\bar{n}_{c}=1, \bar{n}_{h}=2$ (green), and $\bar{n}_{c}=1$, $\bar{n}_{h}=3$ (blue), and we have defined $\gamma \equiv \gamma_{h}+\gamma_{c}$. The dashed lines are approximations based on the branch-points of the cumulant generating function in Eq. (10). At low temperatures, the left line is of the form $\propto \beta_{c} \hbar \omega_{0} J$, while the right one is given by $\propto-\beta_{h} \hbar \omega_{0} J$.

heat flow through the cavity in a nonequilibrium situation [61-64]. Similar to Eq. (3), we can evaluate the MGF at finite times for the transfer of photons between the cavity and each reservoir [65]. Here we are particularly interested in the long-time statistics of the photon current $J$ running via the cavity from a hot to a cold reservoir. For the net photon current, the cumulant generating functions reads

$$
\Theta(s)=\frac{\gamma_{c}+\gamma_{h}}{2}\left(1-\sqrt{1-4 \frac{\gamma_{c} \gamma_{h}}{\left(\gamma_{c}+\gamma_{h}\right)^{2}} \kappa(s)}\right),
$$

with $\quad \kappa(s) \equiv\left(e^{s}-1\right)\left(1+\bar{n}_{c}\right) \bar{n}_{h}+\left(e^{-s}-1\right) \bar{n}_{c}\left(1+\bar{n}_{h}\right)$, where $\bar{n}_{h(c)}$ is the Bose-Einstein distribution of the hot (cold) bath at the photon frequency $\omega_{0}$, and $\gamma_{h(c)}$ is the coupling strength (see Appendix F). This expression also holds for the heat exchange between two resistors connected via a narrow transmission profile [66]. Again, we can evaluate the large-deviation statistics by analytically solving the saddle-point equation. The cumulant generating function has square-root singularities both for positive and negative values of $s$, which determine the linear parts of the large-deviation function for large (positive or negative) photon currents as illustrated in Fig. 3(b). These results resemble the numerical findings of Ref. [41].

\section{FLUCTUATION RELATIONS}

It is interesting to understand the properties of the heat current fluctuations. The cumulant generating function fulfills the symmetry $\Theta(s)=\Theta(-s-\sigma)$, where $\sigma=\hbar \omega_{0}\left(\beta_{c}-\beta_{h}\right)$ determines the entropy increase per transferred photon. This symmetry immediately implies the fluctuation relation $[67,68]$ (see Appendix G)

$$
\frac{1}{t} \ln \left[\frac{P(J, t)}{P(-J, t)}\right]=\sigma J
$$

which connects the probabilities to observe photon currents $J$ of opposite signs, also far from equilibrium with large temperature differences. Close to equilibrium, we may expand the mean heat current $\left\langle J_{Q}\right\rangle \equiv \hbar \omega_{0}\langle J\rangle \simeq G_{Q}^{(1)} \Delta T+G_{Q}^{(2)} \Delta T^{2} / 2$ and the noise $S_{Q}=\left\langle\left\langle J_{Q}^{2}\right\rangle\right\rangle \simeq S_{Q}^{(\text {eq })}+S_{Q}^{(1)} \Delta T$ in the temperature difference $\Delta T$. From the symmetry of the generating function, we then obtain the fluctuation-dissipation theorem for heat currents, $S_{Q}^{(\mathrm{eq})}=2 k_{B} T^{2} G_{Q}^{(1)}$, relating the equilibrium noise to the linear thermal conductance $[41,69]$. Moreover, we find the relation $S_{Q}^{(1)}=k_{B} T^{2} G_{Q}^{(2)}$ between the noise susceptibility and the second-order response coefficient of the heat current in the weakly nonlinear regime (see Appendix $\mathrm{H}$ ).

\section{NOISE POWER SPECTRUM}

Finally, we turn to the noise spectra of the heat currents. The finite-frequency noise can be obtained from the MGF at finite times using MacDonald's formula [70-72]. In equilibrium, the auto-correlation functions read (see Appendix I)

$$
S_{Q}^{c, h}(\omega)=S_{Q}^{(\mathrm{eq})}\left(1+\frac{\gamma_{c, h}}{\gamma_{h, c}} \frac{\omega^{2}}{\left(\gamma_{c}+\gamma_{h}\right)^{2}+\omega^{2}}\right),
$$

while for the real-part of the cross-correlator, we find

$$
\operatorname{Re}\left[S_{Q}^{c h}(\omega)\right]=S_{Q}^{(\mathrm{eq})}\left(-1+\frac{\omega^{2}}{\left(\gamma_{c}+\gamma_{h}\right)^{2}+\omega^{2}}\right) .
$$

We see that $S_{Q}^{c}(0)=S_{Q}^{h}(0)=-\operatorname{Re}\left[S_{Q}^{c h}(0)\right]=S_{Q}^{(\mathrm{eq})}$, since there is no accumulation of photons in the cavity at low frequencies. Generally, we do not expect simple fluctuationdissipation theorems for the individual heat currents at finite frequencies [73]. On the other hand, using the continuity equation $\dot{U}(t)=-\left[J_{Q}^{c}(t)+J_{Q}^{h}(t)\right]$ for the cavity energy and the outgoing heat currents, we can write the energy fluctuations as $\omega^{2} S_{U}(\omega)=S_{Q}^{c}(\omega)+S_{Q}^{h}(\omega)+2 \operatorname{Re}\left[S_{Q}^{c h}(\omega)\right]$. Now, applying a weak perturbation $\hat{H}^{\prime}(t)=\mathcal{K}(t) \hat{H}$, the change of the cavity energy $\langle\Delta U\rangle(\omega)=\chi(\omega) \mathcal{K}(\omega)$ in the Fourier domain can be expressed in terms of the susceptibility $\chi(\omega)$ in response to the force $\mathcal{K}(\omega)$ [74]. We then arrive at the fluctuation-dissipation theorem, $S_{U}(\omega)=2 k_{B} T \operatorname{Im}[\chi(\omega)] / \omega$, which is valid for frequencies below the temperature, $\hbar \omega \ll k_{B} T$. Combining these expressions brings us to the relation

$$
S_{Q}^{c}(\omega)+S_{Q}^{h}(\omega)+2 \operatorname{Re}\left[S_{Q}^{c h}(\omega)\right]=2 k_{B} T \omega \operatorname{Im}[\chi(\omega)]
$$

between the sum of the noise spectra and the response of the system to small perturbations of the cavity frequency. We expect this relation to hold for many systems, where external reservoirs exchange heat via a central region.

\section{CONCLUSIONS}

We have fully determined the photon counting statistics of a quantum harmonic oscillator with dissipative Lindblad dynamics. To be specific, we have formulated our finding in terms of a microwave cavity, although our general results are valid for any quantum harmonic oscillator. The short-time physics can be characterized by the distribution of photon waiting times, which contains information about few-photon processes that cannot easily be extracted from standard correlation measurements. The factorial cumulants are positive 
at all times, unlike the case of noninteracting electrons for which the sign alternates with the order. This finding indicates that the quantum statistics of the particles, being bosons or fermions, determines the sign of the factorial cumulants. We have obtained a simple expression for the large-deviation statistics of the photon current, which may explain earlier results on heat-transport fluctuations and measurements of work distributions. Finally, we have generalized our problem to a nonequilibrium situation in which a temperature gradient drives a heat current through the cavity. In this case, we have derived fluctuation-dissipation theorems in the linear and weakly nonlinear regimes and formulated a relation between the heat noise spectra and the response of the system to small perturbations of the cavity frequency. These predictions may be tested in future experiments with single-photon detectors or calorimetric measurements of heat currents.

\section{ACKNOWLEDGMENTS}

We thank K. Brandner, A. A. Clerk, F. Hassler, V. F. Maisi, P. P. Potts, and P. Samuelsson for useful discussions. The work was supported by the Swedish Research Council and the Academy of Finland (Projects No. 308515 and No. 312299).

\section{APPENDIX A: FROM THE LINDBLAD EQUATION [Eq. (1)] TO THE PARTIAL DIFFERENTIAL EQUATION Eq. (2)}

We here derive the partial differential equation in Eq. (2) from the Lindblad equation [Eq. (1)] for a single light mode, with resonance frequency $\omega_{0}$, coupled to a single heat bath at temperature $T$ (we consider multiple baths in Appendix F). The light mode is modeled as a quantum harmonic oscillator with the Hamiltonian

$$
\hat{H}=\hbar \omega_{0}\left(\hat{a}^{\dagger} \hat{a}+\frac{1}{2}\right),
$$

where $\hbar$ is the reduced Planck constant and $\hat{a}\left(\hat{a}^{\dagger}\right)$ is the anniliation (creation) operator of the oscillator. Taking the coupling to the heat bath into account, the time evolution of the reduced density matrix $\hat{\rho}$ of the cavity is given by the Lindblad master equation $[43,75,76]$

$$
\begin{aligned}
\frac{d \hat{\rho}}{d t}= & -\frac{i}{\hbar}[\hat{H}, \hat{\rho}]+\gamma(\bar{n}+1)\left(\hat{a} \hat{\rho} \hat{a}^{\dagger}-\frac{1}{2}\left\{\hat{a}^{\dagger} \hat{a}, \hat{\rho}\right\}\right) \\
& +\gamma \bar{n}\left(\hat{a}^{\dagger} \hat{\rho} \hat{a}-\frac{1}{2}\left\{\hat{a} \hat{a}^{\dagger}, \hat{\rho}\right\}\right),
\end{aligned}
$$

which is the same as Eq. (1) in the main text. Here, $\gamma$ is a reference rate of relaxations and excitations in the system induced by the reservoir and

$$
\bar{n} \equiv \frac{1}{e^{\beta \hbar \omega_{0}}-1}
$$

is the average occupation of the light mode in equilibrium at the inverse temperature $\beta \equiv 1 /\left(k_{B} T\right)$.

The Lindblad Eq. (A2) is not dependent on the microscopic details of the heat bath and can describe both bosonic and fermionic heat baths. To see this, we may rewrite the emission and absorption rates as

$$
\gamma(\bar{n}+1)=\gamma \int \frac{d E}{\hbar \omega_{0}} f(E)\left[1-f\left(E+\hbar \omega_{0}\right)\right]
$$

and

$$
\gamma \bar{n}=\gamma \int \frac{d E}{\hbar \omega_{0}} f(E)\left[1-f\left(E-\hbar \omega_{0}\right)\right],
$$

using the definitions of the Bose-Einstein and Fermi-Dirac distributions, $\bar{n}$ and $f(E)=1 /\left(e^{\beta E}+1\right)$. We may think of the left-hand side of these equations as corresponding to a bosonic bath, such as the thermal background radiation, with $\bar{n}$ being the average number of bosons in the reservoir with energy $\hbar \omega_{0}$. Similarly, we may think of the right-hand sides in terms of a fermionic bath, such as the Fermi sea of electrons in a nanoscale conductor. In this case, the emission of a photon from the cavity with energy $\hbar \omega_{0}$ is associated with the excitation of an electron with energy $E$ to a higher-lying state with energy $E+\hbar \omega_{0}$. The absorption of a photon is in a similar manner associated with an electron relaxing from energy $E$ to a lower-lying state with energy $E-\hbar \omega_{0}$. The electronic processes take place close to the Fermi level, $E_{F}=$ 0 , where the electronic density of states is approximately constant, $g(E) \propto 1 /\left(\hbar \omega_{0}\right)$.

\section{Unraveling the master equation}

To keep track of the number $m$ of photons emitted into the heat bath, we introduce the $m$-resolved density matrices $\hat{\rho}(m, t)$, so that $P(m, t)=\operatorname{Tr} \hat{\rho}(m, t)$ is the probability of having emitted $m$ photons to the heat bath. They satisfy the unraveled Lindblad equation [44],

$$
\begin{aligned}
\frac{d \hat{\rho}(m, t)}{d t}= & -\frac{i}{\hbar}[\hat{H}, \hat{\rho}(m, t)] \\
& +\gamma(\bar{n}+1)\left(\hat{a} \hat{\rho}(m-1, t) \hat{a}^{\dagger}-\frac{1}{2}\left\{\hat{a}^{\dagger} \hat{a}, \hat{\rho}(m, t)\right\}\right) \\
& +\gamma \bar{n}\left(\hat{a}^{\dagger} \hat{\rho}(m, t) \hat{a}-\frac{1}{2}\left\{\hat{a} \hat{a}^{\dagger}, \hat{\rho}(m, t)\right\}\right), \quad \text { (A6) }
\end{aligned}
$$

since the gain term $\mathcal{J}_{e} \hat{\rho}=\gamma(\bar{n}+1) \hat{a} \hat{\rho} \hat{a}^{\dagger}$ in the Lindblad equation [Eq. (A2)] is responsible for photon emissions.

Following the framework of full counting statistics [77-79], we introduce a counting field $s$ by performing a Laplace transformation,

$$
\hat{\rho}(s, t) \equiv \sum_{m=0}^{\infty} \hat{\rho}(m, t) e^{m s},
$$

which finally transforms the Lindblad equation to

$$
\begin{aligned}
\frac{d \hat{\rho}(s, t)}{d t}= & -\frac{i}{\hbar}[\hat{H}, \hat{\rho}(s, t)] \\
& +\gamma(\bar{n}+1)\left(e^{s} \hat{a} \hat{\rho}(s, t) \hat{a}^{\dagger}-\frac{1}{2}\left\{\hat{a}^{\dagger} \hat{a}, \hat{\rho}(s, t)\right\}\right) \\
& +\gamma \bar{n}\left(\hat{a}^{\dagger} \hat{\rho}(s, t) \hat{a}-\frac{1}{2}\left\{\hat{a} \hat{a}^{\dagger}, \hat{\rho}(s, t)\right\}\right) .
\end{aligned}
$$

Since we start counting the emitted photons at time $t=0$, the initial probabilities are $P(m, 0)=\delta_{m, 0}$, which translates to the initial condition $\hat{\rho}(s, 0)=\hat{\rho}_{0}$ for the density matrix. 


\section{The generating function $\mathcal{G}(s, q, t)$}

Taking the matrix elements $\left\langle n_{1}|\cdots| n_{2}\right\rangle$ of Eq. (A8), we obtain a set of dynamical equations for the populations $\langle n|\hat{\rho}(s, t)| n\rangle$ and the coherences $\left\langle n_{1}|\hat{\rho}(s, t)| n_{2}\right\rangle\left(n_{1} \neq n_{2}\right)$ of the density matrix. To determine the emission probabilities $P(m, t)$, it is sufficient to determine the dynamics of the populations only, which is possible because the dynamical equations only couple populations to other populations, but not to coherences. The population dynamics can be fully solved by performing another Laplace transformation,

$$
\mathcal{G}(s, q, t) \equiv \sum_{n=0}^{\infty}\langle n|\hat{\rho}(s, t)| n\rangle e^{n q},
$$

where $q$ is the variable conjugate to the populations, and thus recasting the system of dynamical equations into a single partial differential equation,

$$
\partial_{t} \mathcal{G}(s, q, t)=[f(s, q)+g(q)] \partial_{q} \mathcal{G}(s, q, t)+g(q) \mathcal{G}(s, q, t),
$$

which is Eq. (2) in the main text. For the sake of brevity, we have defined the functions

$$
f(s, q) \equiv \gamma(\bar{n}+1)\left(e^{s-q}-1\right), \quad g(q) \equiv \gamma \bar{n}\left(e^{q}-1\right) .
$$

\section{Thermal equilibrium}

At long times, after bringing the system in contact with the reservoir, the system will assume the thermal equilibrium state $\hat{\rho}_{\mathrm{eq}} \equiv e^{-\beta \hat{H}} / Z$, where $Z \equiv \operatorname{Tr} e^{-\beta \hat{H}}$. The probability $P_{\mathrm{eq}}(n) \equiv$ $\left\langle n\left|\hat{\rho}_{\text {eq }}\right| n\right\rangle$ for the cavity to be populated with $n$ photons is then given by the Boltzmann distribution

$$
\begin{aligned}
P_{\mathrm{eq}}(n) & =\frac{1}{Z} e^{-\beta \hbar \omega_{0}(n+1 / 2)}, \\
Z & =\sum_{n=0}^{\infty} e^{-\beta \hbar \omega_{0}(n+1 / 2)}=\frac{1}{e^{\beta \hbar \omega_{0} / 2}-e^{-\beta \hbar \omega_{0} / 2}} .
\end{aligned}
$$

For later convenience, we also calculate the Laplace transform

$$
\mathcal{G}_{\mathrm{eq}}(q) \equiv \sum_{n=0}^{\infty} P_{\mathrm{eq}}(n) e^{n q}=\frac{1}{1+\bar{n}\left(1-e^{q}\right)} .
$$

\section{APPENDIX B: DERIVATION OF THE MOMENT GENERATING FUNCTION [Eq. (3)]}

The general solution of Eq. (A10) has the form

$$
\mathcal{G}(s, q, t)=\mathcal{G}_{0}(s, q, t) \mathcal{G}_{*}(s, q, t),
$$

where $\mathcal{G}_{0}(s, q, t)$ is the general solution of the homogeneous equation

$$
\partial_{t} \mathcal{G}(s, q, t)=[f(s, q)+g(q)] \partial_{q} \mathcal{G}(s, q, t),
$$

and $\mathcal{G}_{*}(s, q, t)$ is a particular solution of Eq. (A10).

\section{Homogeneous solution}

The homogeneous solution is obtained using the method of characteristics [45]. We find the curves $q_{Q}(s, t)$ along which the solutions $\mathcal{G}_{0}(s, q, t)$ are constant,

$$
\frac{d}{d t} \mathcal{G}_{0}\left[s, q_{Q}(s, t), t\right]=0,
$$

where $Q=q_{Q}(s, 0)$ specifies the initial condition of each curve. The general homogeneous solution is then

$$
\mathcal{G}_{0}(s, q, t)=F[s, Q(s, q, t)],
$$

where $Q(s, q, t)$ is the inverse to the equation $q=q_{Q}(s, t)$ and $F(s, q)$ specifies the initial data.

From the condition Eq. (B3) of characteristics, we obtain the ordinary differential equation

$$
\begin{aligned}
\frac{d q_{Q}}{d t} & =-f\left(s, q_{Q}\right)-g\left(q_{Q}\right) \\
& =\gamma\left[2 \bar{n}+1-\bar{n} e^{q_{Q}}-(\bar{n}+1) e^{s} e^{-q_{Q}}\right]
\end{aligned}
$$

for $q_{Q}(s, t)$, which can be solved by separation of variables:

$$
\begin{aligned}
\gamma t & =\int_{Q}^{q_{Q}} \frac{d x}{2 \bar{n}+1-\bar{n} e^{x}-(\bar{n}+1) e^{s} e^{-x}} \\
& =\left.\frac{1}{\xi} \ln \frac{\xi-1-2 \bar{n}\left(1-e^{x}\right)}{\xi+1+2 \bar{n}\left(1-e^{x}\right)}\right|_{x=Q} ^{x=q_{Q}},
\end{aligned}
$$

where $\xi=\sqrt{1-4 \bar{n}(1+\bar{n})\left(e^{s}-1\right)}$. Solving this result for $Q$, we arrive at the result

$$
\begin{aligned}
Q(s, q, t) & \\
= & \ln \left\{\frac{2 \bar{n}+1}{2 \bar{n}}+\frac{\xi}{2 \bar{n}}\right. \\
& \left.\times \frac{\left(\xi-1-2 \bar{n}\left[1-e^{q}\right]\right)-e^{\xi \gamma t}\left(\xi+1+2 \bar{n}\left[1-e^{q}\right]\right)}{\left(\xi-1-2 \bar{n}\left[1-e^{q}\right]\right)+e^{\xi \gamma t}\left(\xi+1+2 \bar{n}\left[1-e^{q}\right]\right)}\right\},
\end{aligned}
$$

which, together with Eq. (B4), solves the homogeneous equation.

\section{Particular solution}

To find one particular solution, we make the ansatz of a time-independent solution $\mathcal{G}_{*}(s, q, t)=\mathcal{G}_{*}(s, q)$. Plugging the ansatz into Eq. (A10) yields

$$
\begin{aligned}
\ln \mathcal{G}_{*}(s, q)= & \int_{0}^{q} \frac{-g(x) d x}{f(s, x)+g(x)}=-\frac{\gamma}{2} \int_{0}^{q} \frac{d x}{f(s, x)+g(x)} \\
& -\frac{1}{2} \int_{0}^{q} 1 d x-\frac{1}{2} \int_{0}^{q} \frac{d[f(s, x)+g(x)]}{d x} \\
& \times \frac{d x}{f(s, x)+g(x)} \\
= & \left.\frac{\gamma t}{2}\right|_{Q=0}-\frac{1}{2} \ln \frac{e^{q}[f(s, q)+g(q)]}{f(s, 0)+g(0)}
\end{aligned}
$$

where $\gamma t$ is short for the expression in Eq. (B6).

\section{General solution and initial value}

The last remaining step is to express the function $F$ appearing in the homogeneous solution Eq. (B4) in terms of the initial condition $\hat{\rho}_{0}$ or, equivalently, the generating function $\mathcal{G}(s, q, 0)$ (which does not depend on $s$ ). Evaluating Eq. (B1) at time $t=0$, we find that $F$ is given as $F(s, q)=$ 
$\mathcal{G}(s, q, 0) / \mathcal{G}_{*}(s, q)$. Therefore, we can write

$$
\mathcal{G}(s, q, t)=\mathcal{G}(s, Q, 0) \frac{\mathcal{G}_{*}(s, q)}{\mathcal{G}_{*}(s, Q)},
$$

where $Q$ is short for the function $Q(s, q, t)$ given in Eq. (B7). Plugging in Eq. (B8), we obtain the full solution

$$
\mathcal{G}(s, q, t)=\mathcal{G}(s, Q, 0) e^{\gamma t / 2} \sqrt{\frac{e^{Q}[f(s, Q)+g(Q)]}{e^{q}[f(s, q)+g(q)]}} .
$$

\section{Moment generating function}

The moment generating function (MGF) $\mathcal{M}(s, t) \equiv$ $\operatorname{Tr} \hat{\rho}(s, t)=\mathcal{G}(s, 0, t)$ is directly obtained from Eq. (B10). Plugging in $q=0$ gives [here $Q_{0} \equiv Q(s, 0, t)$ ]

$$
Q_{0}=\ln \left\{\frac{2 \bar{n}+1}{2 \bar{n}}-\frac{\xi}{2 \bar{n}} \frac{\xi \sinh \left[\frac{\xi \gamma t}{2}\right]+\cosh \left[\frac{\xi \gamma t}{2}\right]}{\xi \cosh \left[\frac{\xi \gamma t}{2}\right]+\sinh \left[\frac{\xi \gamma t}{2}\right]}\right\}
$$

and

$$
\mathcal{M}(s, t)=\mathcal{G}\left(s, Q_{0}, 0\right) \frac{\xi e^{\gamma t / 2}}{\xi \cosh \left[\frac{\xi \gamma t}{2}\right]+\sinh \left[\frac{\xi \gamma t}{2}\right]},
$$

where $\xi=\sqrt{1-4 \bar{n}(1+\bar{n})\left(e^{s}-1\right)}$ as above.

If the system is initially thermalized, we can plug in $\mathcal{G}(s, q, 0)=\mathcal{G}_{\text {eq }}(q)$ as given in Eq. (A13) and readily obtain

$$
\mathcal{M}(s, t)=\frac{2 \xi e^{\gamma t / 2}}{2 \xi \cosh \left[\frac{\xi \gamma t}{2}\right]+\left(1+\xi^{2}\right) \sinh \left[\frac{\xi \gamma t}{2}\right]} .
$$

This equation is identical to Eq. (3) in the main text.

From the MGF, we can, for example, determine the average emission current $\left\langle J_{e}\right\rangle=\left.\partial_{s} \mathcal{M}(s, t)\right|_{s=0} / t$. Using $\xi=1-$ $2 \bar{n}(1+\bar{n}) s+\mathcal{O}\left(s^{2}\right)$, we expand the MGF in powers of $s$, $\mathcal{M}(s, t)=1+\gamma \bar{n}(1+\bar{n}) s t+\mathcal{O}\left(s^{2}\right)$ and read off

$$
\left\langle J_{e}\right\rangle=\gamma \bar{n}(1+\bar{n}) .
$$

\section{Long-time limit}

For long times $\gamma t \gg 1$, we can approximate $\cosh \left[\frac{\xi \gamma t}{2}\right] \simeq$ $\sinh \left[\frac{\xi \gamma t}{2}\right] \simeq e^{\xi \gamma t / 2}$. Applying this to Eq. (B13) yields the MGF in the long-time limit,

$$
\mathcal{M}(s, t) \simeq \frac{4 \xi}{(1+\xi)^{2}} e^{-\gamma t(\xi-1) / 2},
$$

as well as the cumulant generating function:

$$
\begin{aligned}
\Theta(s) & \equiv \lim _{t \rightarrow \infty} \frac{\ln \mathcal{M}(s, t)}{t}=-\frac{\gamma}{2}(\xi-1) \\
& =\frac{\gamma}{2}\left(1-\sqrt{1-4\left(e^{s}-1\right) \bar{n}(1+\bar{n})}\right) .
\end{aligned}
$$

\section{APPENDIX C: UNDERSTANDING THE WAITING TIME DISTRIBUTION [Eq. (4)] WITH BAYES' THEOREM}

We use the MGF in Eq. (B13) to calculate the waiting time distribution (WTD),

$$
\mathcal{W}(\tau)=\langle\tau\rangle \partial_{\tau}^{2} \Pi(\tau)
$$

where $\langle\tau\rangle=1 /\left\langle J_{e}\right\rangle$ is the mean waiting time and $\Pi(\tau)=$ $\mathcal{M}(-\infty, t)=P(n=0, t)$ is the so-called idle-time probability. With $\left\langle J_{e}\right\rangle=\gamma \bar{n}(1+\bar{n}) \equiv \Gamma$, we obtain

$$
\mathcal{W}(\tau)=\Gamma \gamma \bar{\gamma} \frac{\gamma+6 \Gamma+(\gamma+2 \Gamma) \cosh [\bar{\gamma} \tau]+\bar{\gamma} \sinh [\bar{\gamma} \tau]}{\left(\bar{\gamma} \cosh \left[\frac{\bar{\gamma} \tau}{2}\right]+(\gamma+2 \Gamma) \sinh \left[\frac{\bar{\gamma} \tau}{2}\right]\right)^{3}} e^{\frac{\gamma \tau}{2}},
$$

for the WTD, where we have defined $\bar{\gamma}=\gamma(1+2 \bar{n})$. This is the same equation as Eq. (4) in the main text.

Expanding the WTD in $\tau$, we find that it equals

$$
\mathcal{W}(\tau)=2 \Gamma e^{-\gamma[6 \bar{n}(\bar{n}+1)+1] \tau / 2}+\mathcal{O}\left(\tau^{2}\right)
$$

for small waiting times. The leading order at long times is

$$
\mathcal{W}(\tau) \simeq \frac{4 \Gamma \gamma \bar{\gamma}}{(\gamma+\bar{\gamma}+2 \Gamma)^{2}} e^{-\gamma \bar{n} \tau} .
$$

\section{Consecutive emissions at $t=0$}

From Eq. (C3), we find that the probability for a second photon emission immediately after the first is

$$
W(0) d \tau=2 \Gamma d \tau .
$$

Note that $\mathcal{W}(0)=\Gamma g^{(2)}(0)$ as shown in Ref. [29], where $g^{(2)}(t)$ is Glauber's second degree of coherence which we will calculate in Appendix D. The functions $\mathcal{W}(\tau)$ and $\Gamma g^{(2)}(\tau)$ do not agree at first order in $\tau$, however.

The finite value at $\tau=0$ stems from the fact that the photon cavity can contain many photons at the same time and thus emit several photons within an arbitrarily short time, without a need for particle absorption in between each event. The WTD does therefore not display the suppression at short times that WTDs typically display for the emission statistics from single fermionic modes, such as a quantum dot with a single resonance level.

In fact, the cavity has an emission rate that is enhanced by a factor of 2 at $\tau=0$ compared to the average emission rate $\left\langle J_{e}\right\rangle=\Gamma$. This enhancement can be understood from Bayes' theorem. The conditional probability $P\left(n \mid E_{0}\right)$ of having $n$ photons in the cavity directly after a photon emission event $E_{0}$ at time $t=0$ is given by

$$
P\left(n \mid E_{0}\right)=P\left(E_{0} \mid n+1\right) \frac{P_{\mathrm{eq}}(n+1)}{\Gamma d t}=\frac{n+1}{\bar{n}} P_{\mathrm{eq}}(n+1),
$$

where $P_{\mathrm{eq}}(n)$ is the probability for the cavity to be filled with $n$ photons in thermal equilibrium given in Eq. (A12). We here used that the probability $P\left(E_{0} \mid n+1\right)$ for the emission event $E_{0}$, given that there are $n+1$ photons in the cavity just before, is

$$
\begin{aligned}
P\left(E_{0} \mid n+1\right) & =\gamma(\bar{n}+1) \operatorname{Tr}\left[a|n+1\rangle\langle n+1| a^{\dagger}\right] d t \\
& =\gamma(\bar{n}+1)(n+1) d t .
\end{aligned}
$$

From Eq. (C6), it follows that the expected number of photons in the cavity after an emission event is exactly twice as large compared to the steady state:

$$
\sum_{n=0}^{\infty} n P\left(n \mid E_{0}\right)=2 \bar{n}
$$


This result explains our previous observation of the emission rate enhancement, since the emission rate can be calculated as

$$
\Gamma_{c}(0)=\sum_{n=0}^{\infty} \gamma(\bar{n}+1) n P\left(n \mid E_{0}\right)=2 \Gamma,
$$

where, as in the main text, $\Gamma_{c}(\tau)$ is the conditional emission rate at a time $\tau$ after the last emission.

\section{Conditional emission at finite times}

The conditional emission rate $\Gamma_{c}(\tau)$ is generally defined as the conditioned rate for emission events $E_{\tau}$ at time $t=\tau$, given that there was an emission event $E_{0}$ at time $t=0$ and no other emissions in between. By the Kolmogorov definition of conditional probability, this means that

$$
\Gamma_{c}(\tau)=\frac{\mathcal{W}(\tau)}{1-\int_{0}^{\tau} \mathcal{W}(u) d u} .
$$

As shown in Fig. 2(c) in the main text, the emission rate exhibits an enhancement at short times and a suppression at long times compared to its average value $\Gamma$.

From this emission rate, we can calculate the conditional probabilities $P\left(n \mid E_{0}, \tau\right)$ of having $n$ photons in the cavity at a time $\tau$ after the last emission event. The probabilities $P\left(n \mid E_{0}, 0\right)$ are equal to the previously derived $P\left(n \mid E_{0}\right)$, see Eq. (C6). To find the dynamics of these probabilities, we again use the definition of conditional probability, obtaining

$$
P\left(n \mid E_{0}, \tau+d \tau\right)=\frac{\Pi_{n}\left(d \tau \mid E_{0}, \tau\right)}{\Pi\left(d \tau \mid E_{0}, \tau\right)} .
$$

Here $\Pi\left(d \tau \mid E_{0}, \tau\right)=1-\Gamma_{c}(\tau) d \tau$ is the conditioned idletime probability for no emission events during the time $d \tau$, given the emission event $E_{0}$ and that there was no other emission between times $t=0$ and $t=\tau$. The quantity $\Pi_{n}\left(d \tau \mid E_{0}, \tau\right)$ is the conditioned idle-time probability for no emission events during $d \tau$ and for the cavity to contain $n$ photons at time $t=\tau+d \tau$. It can be calculated as

$$
\begin{aligned}
\Pi_{n}\left(d \tau \mid E_{0}, \tau\right)= & P\left(n \mid E_{0}, \tau\right)\left[1-\gamma_{n \rightarrow n+1} d \tau-\gamma_{n \rightarrow n-1} d \tau\right] \\
& +P\left(n-1 \mid E_{0}, \tau\right) \gamma_{n-1 \rightarrow n} d \tau, \quad(\mathrm{C} 12)
\end{aligned}
$$

since $d \tau$ is an infinitesimally short time and there can be at most only one emission or absorption event during this time. Here $\gamma_{n \rightarrow n^{\prime}}$ denotes the rate at which the number of cavity photons changes from $n$ to $n^{\prime}$. More specifically, $\gamma_{n \rightarrow n+1}=$ $\gamma \bar{n}(1+n)$ and $\gamma_{n \rightarrow n-1}=\gamma(\bar{n}+1) n$. Together, we obtain a system

$$
\begin{aligned}
\partial_{\tau} P\left(n \mid E_{0}, \tau\right)= & \Gamma_{c}(\tau) P\left(n \mid E_{0}, \tau\right)+\gamma \bar{n} n P\left(n-1 \mid E_{0}, \tau\right) \\
& -\gamma[\bar{n}(1+n)+(\bar{n}+1) n] P\left(n \mid E_{0}, \tau\right)
\end{aligned}
$$

of differential equations for the conditional probabilities that can be solved at least numerically, see Fig. 2(d). As a consistency check, we can calculate $\Gamma_{c}(\tau)$ as well as the WTD $\mathcal{W}(\tau)$ from the probabilities $P\left(n \mid E_{0}, \tau\right)$ and get back the previous results:

$$
\begin{gathered}
\Gamma_{c}(\tau)=\sum_{n=0}^{\infty} \gamma(\bar{n}+1) n P\left(n \mid E_{0}, \tau\right), \\
\mathcal{W}(\tau)=e^{-\int_{0}^{\tau} \Gamma_{c}(u) d u} \Gamma_{c}(\tau) .
\end{gathered}
$$

\section{Long-time behavior}

In the long-time limit, we expect the probability distribution to reach a steady state. Looking for such a solution, we set $\partial_{\tau} P\left(n \mid E_{0}, \tau\right)$ to zero in Eq. (C13) and obtain

$$
\frac{P_{\mathrm{eq}}\left(n \mid E_{0}, \tau\right)}{P_{\mathrm{eq}}\left(n-1 \mid E_{0}, \tau\right)}=\frac{\bar{n}}{1+2 \bar{n}}
$$

for the steady state. We used that the waiting time distribution for long times [see Eq. (C4)] resembles a Poissonian process with emission rate $\gamma \bar{n}$,

$$
\lim _{\tau \rightarrow \infty} \Gamma_{c}(\tau)=\gamma \bar{n} .
$$

Noting that for a Boltzmann distribution $\frac{P_{\mathrm{eq}}(n)}{P_{\mathrm{eq}}(n-1)}=\frac{\bar{n}}{1+\bar{n}}$, we find that the conditional probability distribution for long times is a Boltzmann distribution as well, albeit with modified $\tilde{n}=$ $\frac{\bar{n}}{1+\bar{n}}$. In other words, $\tilde{n}$ is the expected number of photons in the cavity a long time after the last emission event. As one would expect, $\tilde{n}$ is always between 0 (for $\bar{n}=0$ ) and 1 (for $\bar{n} \rightarrow \infty$ ): If there has not been any emission for a very long time, we expect the average number of photons in the cavity to be less than one. Note also that $\tilde{n} \approx \bar{n}$ for low temperatures.

\section{APPENDIX D: CALCULATION OF THE $g^{(2)}$-FUNCTION} [Eq. (5)]

From the MGF in Eq. (B13), we calculate the noise spectrum $S(\omega)$ of the emission current using MacDonald's formula [70],

$$
S(\omega)=\omega \int_{0}^{\infty} d t \sin (\omega t) \frac{d}{d t}\left\langle\left\langle m^{2}\right\rangle\right\rangle(t)=\Gamma\left(1+2 \frac{\Gamma \gamma}{\gamma^{2}+\omega^{2}}\right),
$$

where

$$
\begin{aligned}
\left\langle\left\langle m^{2}\right\rangle\right\rangle(t)= & \left.\frac{d^{2}}{d s^{2}} \mathcal{M}(s, t)\right|_{s=0}=\bar{n}(1+\bar{n})\left[2 \bar{n}(1+\bar{n}) e^{-\gamma t}\right. \\
& +\gamma t+\bar{n}(1+\bar{n})(\gamma t[2+\gamma t]-2)] .
\end{aligned}
$$

The first part in Eq. (D1) is due to self-correlations. From the definitions of $S(\omega)$ and $g^{(2)}(\tau)$, it follows that [56] $g^{(2)}(\tau)$ is related to the inverse Fourier transform of the noise spectrum without the self-correlation part as

$$
g^{(2)}(\tau)=1+\frac{1}{2 \pi \Gamma^{2}} \int_{-\infty}^{\infty} d \omega e^{-i \omega \tau}[S(\omega)-\Gamma]=1+e^{-\gamma|\tau|},
$$

which provides the result given in Eq. (5) in the main text. An important observation is that the $g^{(2)}$-function does not depend on the bath temperature. Therefore, it cannot be possible, in general, to derive the waiting time distribution from the $g^{(2)}$ function alone.

\section{Photon emission is not a renewal process}

For renewal processes, for which subsequent waiting times are uncorrelated, the WTD can be derived from the $g^{(2)}$ function and $\Gamma=\left\langle J_{e}\right\rangle$ using the relation

$$
\Gamma g^{(2)}(s)=\mathcal{W}(s) /[1-\mathcal{W}(s)]
$$

for the Laplace transformed $g^{(2)}(s)$ and $\mathcal{W}(s)$ [29]. 
Here, we compute the WTD $\mathcal{W}_{\text {re }}(\tau)$ that one obtains from applying this formula to the present case, therefore assuming that the photon emission process is a renewal process. We show that this distribution is different from the correct WTD given in Eq. (C2), thus showing explicitly that the process is not a renewal process.

The Laplace transform of the $g^{(2)}$-function given in Eq. (D3) is $g^{(2)}(s)=\frac{1}{s}+\frac{1}{s+\gamma}$, using Eq. (D4) we get

$$
\mathcal{W}_{\mathrm{re}}(s)=\frac{\Gamma g^{(2)}(s)}{1+\Gamma g^{(2)}(s)}=\frac{\Gamma(2 s+\gamma)}{s(s+\gamma)+\Gamma(2 s+\gamma)} .
$$

Performing an inverse Laplace transform, we obtain

$$
\mathcal{W}_{\mathrm{re}}(\tau)=\frac{\Gamma}{\tilde{\Gamma}}\left[\tilde{\Gamma}\left(e^{\tilde{\Gamma} \tau}+1\right)-2 \Gamma\left(e^{\tilde{\Gamma} \tau}-1\right)\right] e^{-\frac{1}{2}(\gamma+2 \Gamma+\tilde{\Gamma}) \tau},
$$

with $\tilde{\Gamma}=\sqrt{\gamma^{2}+4 \Gamma^{2}}=\gamma \sqrt{1+4 n^{2}(1+n)^{2}}$. This WTD is evidently different from the one given in Eq. (C2), showing that the emission statistics is a nonrenewal process. At short waiting times $\tau$, the expression Eq. (D6) reduces to

$$
\mathcal{W}_{\mathrm{re}}(\tau) \simeq 2 \Gamma e^{-\gamma[4 \bar{n}(\bar{n}+1)+1] \tau / 2},
$$

which can be compared to the short-time WTD given in Eq. (C3), $\mathcal{W}(\tau) \simeq 2 \Gamma e^{-\gamma[6 \bar{n}(\bar{n}+1)+1] \tau / 2}$. The WTD of the cavity decays faster as a result of the bunching effect. We see that for low temperatures, for which $\bar{n} \rightarrow 0$, the two distributions give the same result. In that case, it is very unlikely that there is more than one photon in the cavity and thus the cavity returns to the same state after every emission; this is a renewal process.

\section{APPENDIX E: LARGE-DEVIATION STATISTICS OF THE EMISSION CURRENT}

Here we discuss the long-time statistics of the emission current, described by the cumulant generating function $\Theta(s) \equiv \lim _{t \rightarrow \infty} \frac{\ln \mathcal{M}(s, t)}{t}$. As shown in Appendix B, it has the form

$$
\Theta(s)=\frac{\gamma}{2}\left(1-\sqrt{1-4\left(e^{s}-1\right) \bar{n}(1+\bar{n})}\right)
$$

for the emission current. This is the same equation as Eq. (7) in the main text.

We recall that the MGF $\mathcal{M}(s, t) \sim e^{\Theta(s) t}$ is defined as

$$
\mathcal{M}(s, t)=\sum_{m} P(m, t) e^{m s},
$$

where $P(m, t)$ is the probability to have emitted $m$ photons at time $t$. This relation allows us to extract the probability $P\left(J_{e}, t\right)$ for having an average emission current $J_{e}=m / t$ during a measurement time $t$ as a Fourier coefficient of the MGF; it is

$$
\begin{aligned}
P\left(J_{e}, t\right) & =\frac{1}{2 \pi i} \int_{-i \pi}^{i \pi} d s \mathcal{M}(s, t) e^{-m s} \\
& =\frac{1}{2 \pi i} \int_{-i \pi}^{i \pi} d s e^{t\left[\Theta(s)-s J_{e}\right]} .
\end{aligned}
$$

In the long-time limit, this integral can be solved using the saddle-point approximation. Let $s_{0}$ be the solution to the saddle-point equation,

$$
\Theta^{\prime}\left(s_{0}\right)=J_{e},
$$

then the exponent of the integral equals $t\left[\Theta\left(s_{0}\right)-s_{0} J_{e}+\right.$ $\left.\frac{1}{2} \Theta^{\prime \prime}\left(s_{0}\right)\left(s-s_{0}\right)^{2}\right]$ to second order. The integral can be performed explicitly, and after taking the limit of large $t$ we are only left with

$$
\frac{\ln \left[P\left(J_{e}, t\right)\right]}{t} \simeq \Theta\left(s_{0}\right)-s_{0} J_{e}
$$

up to terms of order $\ln [t] / t$, note that $s_{0}$ does not depend on $t$. This is Eq. (8) in the main text. The quantity $\lim _{t \rightarrow \infty} \ln \left[P\left(J_{e}, t\right)\right] / t$ is called the large deviation function.

Solving Eq. (E4), we obtain

$$
s_{0}=\ln \left[\frac{J_{e}}{\gamma \Gamma}\left(\sqrt{4 J_{e}^{2}+\gamma^{2}+4 \gamma \Gamma}-2 J_{e}\right)\right],
$$

and plugging this back into Eq. (E5) gives the final result:

$$
\begin{aligned}
\frac{\ln \left[P\left(J_{e}, t\right)\right]}{t}= & \frac{\gamma}{2}+J_{e}-\frac{1}{2} \sqrt{4 J_{e}^{2}+\gamma^{2}+4 \gamma \Gamma} \\
& +J_{e} \ln \left[\frac{\gamma \Gamma}{J_{e}\left(\sqrt{4 J_{e}^{2}+\gamma^{2}+4 \gamma \Gamma}-2 J_{e}\right)}\right] .
\end{aligned}
$$

For examples illustrating this distribution, see Fig. 3(a).

\section{Large $J_{e}$ limit}

For $J_{e} \gg \gamma, \Gamma$, we obtain from Eq. (E6) that

$$
s_{0} \simeq s_{c} \equiv \ln \left[1+\frac{\gamma}{4 \Gamma}\right],
$$

where $s_{c}$ is the locus of the square-root singularity of the cumulant-generating function $\Theta(s)$ with $\Theta\left(s_{c}\right)=\gamma / 2$. Plugging back into Eq. (E5), we obtain

$$
\frac{\ln \left[P\left(J_{e}, t\right)\right]}{t} \simeq \frac{\gamma}{2}-\ln \left[1+\frac{\gamma}{4 \Gamma}\right] J_{e} .
$$

Thus the tail of the probability distribution decays exponentially.

In the limit of high or low temperatures, this expression can be simplified further. For high temperatures $\bar{n} \gtrsim$ 1 , we approximate the slope as $\ln \left[1+\frac{\gamma}{4 \Gamma}\right] \approx\left(\beta \hbar \omega_{0}\right)^{2} / 4$, resulting in

$$
\frac{\ln \left[P\left(J_{e}, t\right)\right]}{t} \simeq \frac{\gamma}{2}-\frac{\left(\beta \hbar \omega_{0}\right)^{2}}{4} J_{e},
$$

whereas for low temperatures $\bar{n} \ll 1$, we use that $\ln \left[1+\frac{\gamma}{4 \Gamma}\right] \approx \beta \hbar \omega_{0}-\ln 4$. At even lower temperatures, we can neglect also the constant offset, obtaining

$$
\frac{\ln \left[P\left(J_{e}, t\right)\right]}{t} \simeq \frac{\gamma}{2}-\beta \hbar \omega_{0} J_{e},
$$

which is Eq. (9) in the main text.

\section{Poissonian limit}

In the other limit, $J_{e} \ll \gamma$, we obtain from Eq. (E7)

$$
\frac{\ln \left[P\left(J_{e}, t\right)\right]}{t} \simeq\left(J_{e}-\gamma \bar{n}\right)-J_{e} \ln \left[J_{e} \frac{\bar{\gamma}}{\gamma \Gamma}\right] .
$$


For $\bar{n} \ll 1$, this is a Poissonian distribution corresponding to the CGF

$$
\Theta_{\text {poiss }}(s)=\Gamma\left(e^{s}-1\right),
$$

which is exactly what Eq. (E1) reduces to in this limit.

\section{APPENDIX F: GENERALIZATION TO MULTIPLE HEAT BATHS}

Here we generalize the previous results to multiple heat baths. We consider a cavity coupled to $N$ heat baths, each with a coupling constant $\gamma_{i}$ and an inverse temperature $\beta_{i}$. The Lindblad equation is

$$
\begin{aligned}
\frac{d \hat{\rho}}{d t}= & -\frac{i}{\hbar}[\hat{H}, \hat{\rho}]+\sum_{i=1}^{N}\left[\gamma_{i}\left(\bar{n}_{i}+1\right)\left(\hat{a} \hat{\rho} \hat{a}^{\dagger}-\frac{1}{2}\left\{\hat{a}^{\dagger} \hat{a}, \hat{\rho}\right\}\right)\right. \\
& \left.+\gamma_{i} \bar{n}_{i}\left(\hat{a}^{\dagger} \hat{\rho} \hat{a}-\frac{1}{2}\left\{\hat{a} \hat{a}^{\dagger}, \hat{\rho}\right\}\right)\right],
\end{aligned}
$$

which is a direct generalization of Eq. (A2). As before, $\bar{n}_{i}=$

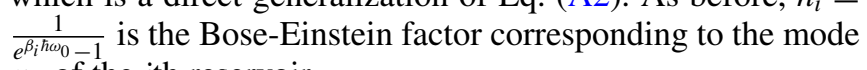
$\omega_{0}$ of the $i$ th reservoir.

To keep track of the number $m_{i}^{+}\left(m_{i}^{-}\right)$of photons emitted into (absorbed from) heat bath $i$, we introduce the $m_{i}^{ \pm}$-resolved density matrices $\hat{\rho}\left(m_{i}^{ \pm}, t\right)$, so that $P\left(m_{i}^{ \pm}, t\right)=$ $\operatorname{Tr} \hat{\rho}\left(m_{i}^{ \pm}, t\right)$ is the probability of having emitted/absorbed $m_{i}^{ \pm}$ photons to/from heat bath $i$. Analogously to the single-bath case, we then perform a Laplace transformation,

$$
\hat{\rho}\left(s_{i}^{ \pm}, t\right)=\sum_{m_{i}^{ \pm}=0}^{\infty} \hat{\rho}\left(m_{i}^{ \pm}, t\right) e^{\sum_{i=1}^{N}\left(m_{i}^{+} s_{i}^{+}-m_{i}^{-} s_{i}^{-}\right)},
$$

with two counting fields per bath, $s_{i}^{+}$for absorption and $s_{i}^{-}$for emission. We then introduce the generating function $\mathcal{G}\left(s_{i}^{ \pm}, q, t\right)=\sum_{n=0}^{\infty}\left\langle n\left|\hat{\rho}\left(s_{i}^{ \pm}, t\right)\right| n\right\rangle e^{n q}$ and obtain the partial differential equation,

$$
\begin{aligned}
\partial_{t} \mathcal{G}\left(s_{i}^{ \pm}, q, t\right)= & \left.f\left(s_{i}^{+}, q\right)+g\left(s_{i}^{-}, q\right)\right] \partial_{q} \mathcal{G}\left(s_{i}^{ \pm}, q, t\right) \\
& +g\left(s_{i}^{-}, q\right) \mathcal{G}\left(s_{i}^{ \pm}, q, t\right),
\end{aligned}
$$

with

$$
f\left(s_{i}^{+}, q\right) \equiv \sum_{i=1}^{N} \gamma_{i}\left(\bar{n}_{i}+1\right)\left(e^{s_{i}^{+}-q}-1\right)
$$

and

$$
g\left(s_{i}^{-}, q\right) \equiv \sum_{i=1}^{N} \gamma_{i} \bar{n}_{i}\left(e^{q-s_{i}^{-}}-1\right) .
$$

From the method of characteristics, we get the solution,

$$
\begin{aligned}
\mathcal{G}\left(s_{i}^{ \pm}, q, t\right) & =\mathcal{G}\left(s_{i}^{ \pm}, Q, 0\right) e^{\gamma_{\Sigma} t / 2} \\
& \times \sqrt{\frac{e^{Q}\left[f\left(s_{i}^{+}, Q\right)+g\left(s_{i}^{-}, Q\right)\right]}{e^{q}\left[f\left(s_{i}^{+}, q\right)+g\left(s_{i}^{-}, q\right)\right]}},
\end{aligned}
$$

with $\gamma_{\Sigma}=\sum_{i=1}^{N} \gamma_{i}$ and

$$
Q(s, q, t)=\ln \left\{\frac{2 \bar{n}+1}{2 n^{-}}+\frac{\xi}{2 n^{-}} \frac{\left.\left(\xi-[1+2 \bar{n}]+2 n^{-} e^{q}\right)-e^{\xi \gamma_{\Sigma} t}\left(\xi+[1+2 \bar{n}]-2 n^{-} e^{q}\right]\right)}{\left.\left(\xi-[1+2 \bar{n}]+2 n^{-} e^{q}\right)+e^{\xi \gamma_{\Sigma} t}\left(\xi+[1+2 \bar{n}]-2 n^{-} e^{q}\right]\right)}\right\}
$$

Similar to before, $\xi$ is defined as $\xi=$ $\sqrt{(2 \bar{n}+1)^{2}-4 n^{-}\left(n^{+}+1\right)}$, where $\bar{n}=\sum_{i=1}^{N} \frac{\gamma_{i}}{\gamma_{\Sigma}} \bar{n}_{i}$ is the average number of photons in the cavity in the steady state, and $n^{-}=\sum_{i=1}^{N} \frac{\gamma_{i}}{\gamma_{\Sigma}} \bar{n}_{i} e^{-s_{i}^{-}}$and $n^{+}+1=\sum_{i=1}^{N} \frac{\gamma_{i}}{\gamma_{\Sigma}}\left(\bar{n}_{i}+1\right) e^{s_{i}^{+}}$. We focus on the case where the initial state is the steady state, i.e., $\mathcal{G}\left(s_{i}^{ \pm}, q, 0\right)=\mathcal{G}_{\text {eq }}(q)$, see Eq. (A13).

\section{Emission current statistics}

To generalize the previous results for the emission statistics, we compute the MGF for photon emission from the cavity to heat bath $i=1$. To this end, we set all counting fields to zero except $s \equiv s_{1}^{+}$, the counting field corresponding to emission into heat bath $i=1$. The $\operatorname{MGF} \mathcal{M}(s, t)=$ $\operatorname{Tr} \hat{\rho}(s, t)=\mathcal{G}(s, 0, t)$ can be calculated from Eq. (F6), it is

$$
\mathcal{M}(s, t)=\frac{2 \xi e^{\gamma_{\Sigma} t / 2}}{2 \xi \cosh \left[\frac{\xi \gamma_{\Sigma} t}{2}\right]+\left(1+\xi^{2}\right) \sinh \left[\frac{\xi \gamma_{\Sigma} t}{2}\right]},
$$

where $\xi=\sqrt{1-4 \frac{\gamma_{1}}{\gamma_{\Sigma}} \bar{n}\left(1+\bar{n}_{1}\right)\left(e^{s}-1\right)}$. We see that the MGF resembles the one of a single heat bath given in Eq. (B13).
From the MGF, we obtain the waiting time distribution,

$$
\begin{aligned}
\mathcal{W}(\tau)= & \Gamma \gamma_{\Sigma} \bar{\gamma} \frac{\gamma_{\Sigma}+6 \Gamma+\left(\gamma_{\Sigma}+2 \Gamma\right) \cosh [\bar{\gamma} \tau]+\bar{\gamma} \sinh [\bar{\gamma} \tau]}{\left(\bar{\gamma} \cosh \left[\frac{\bar{\gamma} \tau}{2}\right]+\left(\gamma_{\Sigma}+2 \Gamma\right) \sinh \left[\frac{\bar{\gamma} \tau}{2}\right]\right)^{3}} \\
& \times e^{\frac{\gamma_{\Sigma} \tau}{2}},
\end{aligned}
$$

with $\bar{\gamma}=\gamma_{\Sigma} \sqrt{1+4 \frac{\gamma_{1}}{\gamma_{\Sigma}} \bar{n}\left(1+\bar{n}_{1}\right)}$. Here, $\Gamma=\gamma_{1} \bar{n}\left(1+n_{1}\right)$ is the average emission rate into the first reservoir and the mean waiting time is $\langle\tau\rangle=\Gamma^{-1}$.

Similarly, we get the $g^{(2)}$-function

$$
g^{(2)}(\tau)=1+e^{-\gamma_{\Sigma}|\tau|},
$$

which, again, is temperature independent in contrast to the WTD.

\section{Net current statistics}

We consider the net current statistics between the cavity and a heat bath with average occupation number $\bar{n}_{c}$ and coupling strength $\gamma_{c}$. The cavity is assumed to be coupled to another heat bath with occupation number $\bar{n}_{h}$ and coupling strength $\gamma_{h}$. The MGF of the net current is obtained as $\mathcal{M}(s, t)=\operatorname{Tr} \hat{\rho}(s, t)=\mathcal{G}(s, 0, t)$ (where $s=s_{c}^{+}=s_{c}^{-}$and 
$\left.s_{h}^{+}=s_{h}^{-}=0\right)$, yielding

$$
\mathcal{M}(s, t)=\frac{2 \xi e^{\gamma_{\Sigma} t / 2}}{2 \xi \cosh \left[\frac{\xi \gamma_{\Sigma} t}{2}\right]+\left(1+\chi^{2}\right) \sinh \left[\frac{\xi \gamma_{\Sigma} t}{2}\right]},
$$

where

$$
\begin{aligned}
& \xi=\sqrt{1-4 \frac{\gamma_{c} \gamma_{h}}{\gamma_{\Sigma}^{2}}\left[\left(e^{s}-1\right)\left(1+\bar{n}_{c}\right) \bar{n}_{h}+\left(e^{-s}-1\right) \bar{n}_{c}\left(1+\bar{n}_{h}\right)\right]}, \\
& \chi=\sqrt{1-4 \frac{\gamma_{c} \gamma_{\Sigma}}{\gamma_{\Sigma}^{2}}\left[\left(e^{s}-1\right)\left(1+\bar{n}_{c}\right) \bar{n}+\left(e^{-s}-1\right) \bar{n}_{c}(1+\bar{n})\right]} .
\end{aligned}
$$

If the temperature of the cold reservoir is very low, the photon current from the cold reservoir into the system goes to zero and this result reduces to the previously derived emission current statistics. More precisely, if $\bar{n}_{c}$ is set to zero in Eq. (F11), we obtain back the MGF in Eq. (B13) describing the emission current into a single heat bath with the decay rate $\gamma=\gamma_{c}+\gamma_{h}$ and an effective temperature given by

$$
\bar{n}_{\mathrm{eff}}=\frac{1}{2}\left(\sqrt{1+4 \bar{n}_{h} \frac{\gamma_{c} \gamma_{h}}{\left(\gamma_{c}+\gamma_{h}\right)^{2}}}-1\right) .
$$

Moreover, for $\gamma_{c} \gg \gamma_{h}$, these expressions simplify to $\gamma \simeq \gamma_{c}$ and $\bar{n}_{\text {eff }} \simeq\left(\gamma_{h} / \gamma_{c}\right) \bar{n}_{h}$.

In the long-time limit, we find the cumulant generating function $\Theta(s)=\lim _{t \rightarrow \infty} \frac{\ln \mathcal{M}(s, t)}{t}$ for the net current,

$$
\Theta(s)=\frac{\gamma_{c}+\gamma_{h}}{2}\left(1-\sqrt{1-4 \frac{\gamma_{c} \gamma_{h}}{\left(\gamma_{c}+\gamma_{h}\right)^{2}} \kappa(s)}\right)
$$

with $\quad \kappa(s) \equiv\left(e^{s}-1\right)\left(1+\bar{n}_{c}\right) \bar{n}_{h}+\left(e^{-s}-1\right) \bar{n}_{c}\left(1+\bar{n}_{h}\right)$, which is Eq. (10) in the main text.

\section{APPENDIX G: DERIVATION OF THE FLUCTUATION RELATION [Eq. (11)]}

To derive a fluctuation relation for the net current in the long-time limit, we note that the CGF in Eq. (F14) fulfills the symmetry property,

$$
\Theta(s)=\Theta(-s-\sigma),
$$

with $\sigma=\hbar \omega_{0}\left(\beta_{c}-\beta_{h}\right)$ determining the entropy increase per transferred photon. We then obtain the following result for the probability distribution:

$$
\begin{aligned}
P(J, t) & =\frac{1}{2 \pi} \int_{-\infty}^{\infty} e^{\Theta(s)} e^{-s J t} d s=\frac{1}{2 \pi} \int_{-\infty}^{\infty} e^{\Theta(-s-\sigma)} e^{-s J t} d s \\
& =\frac{1}{2 \pi} \int_{-\infty}^{\infty} e^{\Theta(s)} e^{(s+\sigma) J t} d s=P(-J, t) e^{\sigma t J} .
\end{aligned}
$$

We have thus derived the fluctuation relation:

$$
\frac{1}{t} \ln \left[\frac{P(J, t)}{P(-J, t)}\right]=\sigma J
$$

This is Eq. (11) in the main text.

\section{APPENDIX H: RELATIONS BETWEEN EQUILIBRIUM NOISE AND RESPONSE COEFFICIENTS}

From the symmetry property $\Theta(s)=\Theta(-s-\sigma)$, we now also derive the fluctuation-dissipation theorem. For clarity, we will below let $\Theta(s, \sigma)$ have a second argument indicating the dimensionless temperature difference $\sigma$ of the cavity. The average particle current between two heat baths with different temperatures, $\sigma /\left(\hbar \omega_{0}\right)=\beta_{c}-\beta_{h}$, is then given by

$$
\begin{aligned}
\langle I\rangle & =\left.\partial_{s} \Theta(s, \sigma)\right|_{s=0}=\left.\partial_{s} \Theta(-s-\sigma, \sigma)\right|_{s=0} \\
& =-\Theta^{(1,0)}(-\sigma, \sigma),
\end{aligned}
$$

where the superscripts refers to the number of derivatives with respect to the first and the second argument, respectively. All quantities are evaluated at $s=0$ after the differentiations. Expanding $\langle I\rangle$ in $\sigma$ to second order, we obtain

$$
\begin{aligned}
\langle I\rangle \approx & -\Theta^{(1,0)}(0,0)+\left[\Theta^{(2,0)}(0,0)-\Theta^{(1,1)}(0,0)\right] \sigma \\
& +\left[-\Theta^{(3,0)}(0,0)+2 \Theta^{(2,1)}(0,0)-\Theta^{(1,2)}(0,0)\right] \frac{\sigma^{2}}{2} .
\end{aligned}
$$

Using that all odd cumulants are zero in equilibrium, $\Theta^{(n, 0)}(0,0)=0$ for $n=1,3,5, \ldots$, and identifying each prefactor of $\sigma^{n} / n$ ! with $\left.\frac{\partial^{n}\langle I\rangle}{\partial \sigma^{n}}\right|_{\mathrm{eq}}=\Theta^{(1, n)}(0,0)$, we obtain the following relations:

$$
\Theta^{(1,1)}(0,0)=\frac{1}{2} \Theta^{(2,0)}(0,0), \quad \Theta^{(1,2)}(0,0)=\Theta^{(2,1)}(0,0) .
$$

\section{Linear regime}

We consider the linear thermal conductance,

$$
\begin{aligned}
\frac{1}{\hbar \omega_{0}} G_{Q}^{(1)} & =\left.\frac{1}{\hbar \omega_{0}} \frac{\partial\langle J\rangle}{\partial \Delta T}\right|_{\Delta T=0}=\left.\left.\frac{\partial\langle I\rangle}{\partial \sigma}\right|_{\sigma=0} \frac{\partial \sigma}{\partial \Delta T}\right|_{\Delta T=0} \\
& =\left.\Theta^{(1,1)}(0,0) \frac{\partial \sigma}{\partial \Delta T}\right|_{\Delta T=0} \\
& =\left.\frac{1}{2} \Theta^{(2,0)}(0,0) \frac{\partial \sigma}{\partial \Delta T}\right|_{\Delta T=0},
\end{aligned}
$$

where $\langle J\rangle=\hbar \omega_{0}\langle I\rangle$ is the heat current. Using that $\left.\frac{\partial \sigma}{\partial \Delta T}\right|_{\Delta T=0}=\frac{\hbar \omega_{0}}{k_{B} T^{2}}$, we obtain

$$
G_{Q}^{(1)}=\left(\hbar \omega_{0}\right)^{2} \frac{1}{2 k_{B} T^{2}} \Theta^{(2,0)}(0,0),
$$

or

$$
S_{Q}^{(\mathrm{eq})}=2 k_{B} T^{2} G_{Q}^{(1)},
$$

where we have introduced the equilibrium heat noise $S_{Q}^{(\mathrm{eq})}=$ $\left(\hbar \omega_{0}\right)^{2} \Theta^{(2,0)}(0,0)$. This is the fluctuation-dissipation theorem for heat currents, relating the equilibrium noise to the linear thermal conductance. 


\section{Weakly nonlinear regime}

For the weakly nonlinear regime, we get

$$
\begin{aligned}
G_{Q}^{(2)} & =\left.\frac{\partial^{2}\langle J\rangle}{\partial \Delta T^{2}}\right|_{\Delta T=0}=\left.\hbar \omega_{0} \frac{\partial^{2}\langle I\rangle}{\partial \Delta T^{2}}\right|_{\Delta T=0}=\left.\hbar \omega_{0}\left(F^{(1,2)}(0,0)\left[\frac{\partial \sigma}{\partial \Delta T}\right]^{2}+F^{(1,1)}(0,0) \frac{\partial^{2} \sigma}{\partial \Delta T^{2}}\right)\right|_{\Delta T=0} \\
& =\left.\hbar \omega_{0}\left(F^{(2,1)}(0,0)\left[\frac{\hbar \omega_{0}}{k_{B} T^{2}}\right]^{2}\right)\right|_{\Delta T=0}=\left.\left.\frac{1}{\hbar \omega_{0}} \frac{\partial S_{Q}}{\partial \Delta T}\right|_{\mathrm{eq}} \frac{\partial \Delta T}{\partial \sigma}\right|_{\Delta T=0}\left[\frac{\hbar \omega_{0}}{k_{B} T^{2}}\right]^{2}=\left.\frac{\partial S_{Q}}{\partial \Delta T}\right|_{\Delta T=0} \frac{1}{k_{B} T^{2}},
\end{aligned}
$$

where we have used $\left.\frac{\partial^{2} \sigma}{\partial \Delta T^{2}}\right|_{\Delta T=0}=0$. We thus arrive at the relation

$$
\left.S_{Q}^{(1)} \equiv \frac{\partial S_{Q}}{\partial \Delta T}\right|_{\mathrm{eq}}=k_{B} T^{2} G_{Q}^{(2)}
$$

\section{APPENDIX I: NOISE POWER SPECTRUM AND THE FLUCTUATION-DISSIPATION THEOREM AT FINITE FREQUENCY}

We now consider a setup with a cavity coupled to two heat baths with the same temperature, i.e., the average photon occupation number is $\bar{n} \equiv \bar{n}_{c}=\bar{n}_{h}$. From the MGF in Eq. (F11), we then obtain

$$
\begin{aligned}
\frac{\left\langle m_{c, h}^{2}\right\rangle(t)}{2 \bar{n}(1+\bar{n})} & =\frac{\gamma_{c, h}^{2}\left[1-e^{-t\left(\gamma_{c}+\gamma_{h}\right)}\right]+t \gamma_{c} \gamma_{h}\left(\gamma_{c}+\gamma_{h}\right)}{\left(\gamma_{c}+\gamma_{h}\right)^{2}}, \\
\frac{\left\langle\left\langle m_{c} m_{h}\right\rangle\right\rangle(t)}{2 \bar{n}(1+\bar{n})} & =\frac{\gamma_{c} \gamma_{h}\left[1-e^{-t\left(\gamma_{c}+\gamma_{h}\right)}-t\left(\gamma_{c}+\gamma_{h}\right)\right]}{\left(\gamma_{c}+\gamma_{h}\right)^{2}},
\end{aligned}
$$

where $m_{c}\left(m_{h}\right)$ denotes the number of particles transferred into heat bath $c(h)$ over a time $t$. Using MacDonald's formula [see Eq. (D1)], we obtain the following expression for the spectral densities $S_{Q}^{c}(\omega), S_{Q}^{h}(\omega)$ and $S_{Q}^{c h}(\omega)$ of the particle currents (to the cold and hot baths and the cross term, respectively),

$$
\begin{aligned}
S_{Q}^{c, h}(\omega) & =S_{Q}^{(\mathrm{eq})}\left(1+\frac{\gamma_{c, h}}{\gamma_{h, c}} \frac{\omega^{2}}{\left(\gamma_{c}+\gamma_{h}\right)^{2}+\omega^{2}}\right), \\
\operatorname{Re}\left[S_{Q}^{c h}(\omega)\right] & =S_{Q}^{(\mathrm{eq})}\left(-1+\frac{\omega^{2}}{\left(\gamma_{c}+\gamma_{h}\right)^{2}+\omega^{2}}\right),
\end{aligned}
$$

where $S_{Q}^{(\mathrm{eq})}=2 \bar{n}(1+\bar{n}) \frac{\gamma_{c} \gamma_{h}}{\gamma_{c}+\gamma_{h}}=2 k_{B} T^{2} G_{Q}^{(1)}$. These equations are identical to Eqs. (12) and (13) in the main text.

Using the continuity equation, $\dot{U}(t)=-\left[J_{Q}^{c}(t)+J_{Q}^{h}(t)\right]$ for the cavity energy and the outgoing heat currents, we write the energy fluctuations as

$$
\omega^{2} S_{U}(\omega)=S_{Q}^{c}(\omega)+S_{Q}^{h}(\omega)+2 \operatorname{Re}\left[S_{Q}^{c h}(\omega)\right] .
$$

From this equation, we get

$$
S_{U}(\omega)=2\left(\hbar \omega_{0}\right)^{2} \bar{n}(1+\bar{n}) \frac{\left(\gamma_{c}+\gamma_{h}\right)}{\left(\gamma_{c}+\gamma_{h}\right)^{2}+\omega^{2}}
$$

\section{Linear response}

We consider a perturbed oscillator, with Hamiltonian $\hat{H}(t)=\hat{H}_{0}+\hat{H}_{1}(t)$, where $\hat{H}_{0}=\hbar \omega_{0}\left(\hat{n}+\frac{1}{2}\right)$ is the unperturbed Hamiltonian and $\hat{H}_{1}(t)=\hat{H}_{0} K(t)$ is a weak perturbation, where $K(t)$ determines the modulation. Below we find the susceptibility that relates the response in the cavity energy $\delta U(t)=\hbar \omega_{0} \delta n(t)$ to the modulation $K(t)$.
To this end, we first introduce the mean number of cavity photons $\langle n(t)\rangle=\sum_{n} n P(n, t)$ as a function of time. From the Lindblad equation we have

$$
\frac{d\langle n(t)\rangle}{d t}=\gamma_{c}\left[n_{c}(t)-\langle n(t)\rangle\right]+\gamma_{h}\left[n_{h}(t)-\langle n(t)\rangle\right]
$$

We consider equal temperatures, $n_{c}(t)=n_{h}(t)=$ $\bar{n}-\frac{\hbar \omega_{0}}{k_{B} T} \bar{n}(1+\bar{n}) K(t)$ to first order in $K(t)$. Introducing $\delta n(t)=\langle n(t)\rangle-\bar{n}$, we get

$$
\frac{d \delta n(t)}{d t}=-\left(\gamma_{c}+\gamma_{h}\right) \delta n(t)-\left(\gamma_{c}+\gamma_{h}\right) \frac{\hbar \omega_{0}}{k_{B} T} \bar{n}(1+\bar{n}) K(t)
$$

In the Fourier domain, this gives

$$
\delta n(\omega)=-\frac{\hbar \omega_{0}}{k_{B} T} \frac{\left(\gamma_{c}+\gamma_{h}\right) \bar{n}(1+\bar{n})}{\gamma_{c}+\gamma_{h}+i \omega} K(\omega)
$$

or

$$
\Delta U(\omega)=\hbar \omega_{0} \delta n(\omega)=-\frac{\left(\hbar \omega_{0}\right)^{2}}{k_{B} T} \frac{\left(\gamma_{c}+\gamma_{h}\right) \bar{n}(1+\bar{n})}{\gamma_{c}+\gamma_{h}+i \omega} K(\omega)
$$

From this, we find the susceptibility:

$$
\chi(\omega)=\frac{\Delta U(\omega)}{K(\omega)}=-\frac{\left(\hbar \omega_{0}\right)^{2}}{k_{B} T} \frac{\left(\gamma_{c}+\gamma_{h}\right) \bar{n}(1+\bar{n})}{\gamma_{c}+\gamma_{h}+i \omega}
$$

In particular, we have

$$
\operatorname{Im}[\chi(\omega)]=\frac{\left(\hbar \omega_{0}\right)^{2}}{k_{B} T} \bar{n}(1+\bar{n}) \frac{\left(\gamma_{c}+\gamma_{h}\right) \omega}{\left(\gamma_{c}+\gamma_{h}\right)^{2}+\omega^{2}}
$$

Comparing Eqs. (I4) and (I10), we then find the fluctuationdissipation theorem

$$
S_{U}(\omega)=2 k_{B} T \frac{\operatorname{Im}[\chi(\omega)]}{\omega},
$$

or

$$
S_{Q}^{c}(\omega)+S_{Q}^{h}(\omega)+2 \operatorname{Re}\left[S_{Q}^{c h}(\omega)\right]=2 k_{B} T \omega \operatorname{Im}[\chi(\omega)],
$$

which is Eq. (14) in the main text. 
[1] A. Zagoskin, Quantum Engineering: Theory and Design of Quantum Coherent Structures (Cambridge University Press, Cambridge, 2011).

[2] C. Lang, D. Bozyigit, C. Eichler, L. Steffen, J. M. Fink, A. A. Abdumalikov, M. Baur, S. Filipp, M. P. da Silva, A. Blais, and A. Wallraff, Observation of Resonant Photon Blockade at Microwave Frequencies using Correlation Function Measurements, Phys. Rev. Lett. 106, 243601 (2011).

[3] A. Delteil, W.-B. Gao, P. Fallahi, J. Miguel-Sanchez, and A. Imamoğlu, Observation of Quantum Jumps of a Single Quantum Dot Spin Using Submicrosecond Single-Shot Optical Readout, Phys. Rev. Lett. 112, 116802 (2014).

[4] A. A. Clerk, F. Marquardt, and J. G. E. Harris, Quantum Measurement of Phonon Shot Noise, Phys. Rev. Lett. 104, 213603 (2010).

[5] J. D. Cohen, S. M. Meenehan, G. S. MacCabe, S. Gröblacher, A. H. Safavi-Naeini, F. Marsili, M. D. Shaw, and O. Painter, Phonon counting and intensity interferometry of a nanomechanical resonator, Nature 520, 522 (2015).

[6] J. Splettstoesser and R. J. Haug, Single-electron control in solid state devices, Phys. Status Solidi B 254, 1770217 (2017).

[7] G. Fève, A. Mahé, J.-M. Berroir, T. Kontos, B. Plaçais, D. C. Glattli, A. Cavanna, B. Etienne, and Y. Jin, An on-demand coherent single-electron source, Science 316, 1169 (2007).

[8] E. Bocquillon, V. Freulon, J.-M Berroir, P. Degiovanni, B. Plaçais, A. Cavanna, Y. Jin, and G. Fève, Coherence and indistinguishability of single electrons emitted by independent sources, Science 339, 1054 (2013).

[9] J. Dubois, T. Jullien, F. Portier, P. Roche, A. Cavanna, Y. Jin, W. Wegscheider, P. Roulleau, and D. C. Glattli, Minimal-excitation states for electron quantum optics using levitons, Nature 502, 659 (2013).

[10] T. Jullien, P. Roulleau, B. Roche, A. Cavanna, Y. Jin, and D. C. Glattli, Quantum tomography of an electron, Nature 514, 603 (2014).

[11] I. Aharonovich, D. Englund, and M. Toth, Solid-state singlephoton emitters, Nat. Photonics 10, 631 (2016).

[12] S. Gustavsson, R. Leturcq, M. Studer, I. Shorubalko, T. Ihn, K. Ensslin, D. C. Driscoll, and A. C. Gossard, Electron counting in quantum dots, Surf. Sci. Rep. 64, 191 (2009).

[13] X. Gu, A. F. Kockum, A. Miranowicz, Y. X. Liu, and F. Nori, Microwave photonics with superconducting quantum circuits, Phys. Rep. 718, 1 (2017).

[14] J. P. Pekola, P. Solinas, A. Shnirman, and D. V. Averin, Calorimetric measurement of work in a quantum system, New J. Phys. 15, 115006 (2013).

[15] S. Gasparinetti, K. L. Viisanen, O.-P. Saira, T. Faivre, M. Arzeo, M. Meschke, and J. P. Pekola, Fast Electron Thermometry for Ultrasensitive Calorimetric Detection, Phys. Rev. Appl. 3, 014007 (2015).

[16] F. Brange, P. Samuelsson, B. Karimi, and J. P. Pekola, Nanoscale quantum calorimetry with electronic temperature fluctuations, Phys. Rev. B 98, 205414 (2018).

[17] Y.-F. Chen, D. Hover, S. Sendelbach, L. Maurer, S. T. Merkel, E. J. Pritchett, F. K. Wilhelm, and R. McDermott, Microwave Photon Counter Based on Josephson Junctions, Phys. Rev. Lett. 107, 217401 (2011).

[18] E. D. Walsh, D. K. Efetov, G.-H. Lee, M. Heuck, J. Crossno, T. A. Ohki, P. Kim, D. Englund, and K. C. Fong, Graphene-
Based Josephson-Junction Single-Photon Detector, Phys. Rev. Appl. 8, 024022 (2017).

[19] J. C. Besse, S. Gasparinetti, M.-C. Collodo, T. Walter, P. Kurpiers, M. Pechal, C. Eichler, and A. Wallraff, Single-Shot Quantum Non-Demolition Detection of Itinerant Microwave Photons, Phys. Rev. X 8, 021003 (2018).

[20] S. Vinjanampathy and J. Anders, Quantum thermodynamics, Cont. Phys. 57, 545 (2016).

[21] H. M. Wiseman and G. J. Milburn, Quantum Measurement and Control (Cambridge University Press, Cambridge, 2009) .

[22] M. A. Nielsen and I. L. Chuang, Quantum Computation and Quantum Information (Cambridge University Press, Cambridge, 2011).

[23] M. Esposito, U. Harbola, and S. Mukamel, Nonequilibrium fluctuations, fluctuation theorems, and counting statistics in quantum systems, Rev. Mod. Phys. 81, 1665 (2009).

[24] Y.-Y. Liu, J. Stehlik, C. Eichler, M. J. Gullans, J. M. Taylor, and J. R. Petta, Semiconductor double quantum dot micromaser, Science 347, 285 (2015).

[25] X. Mi, J. V. Cady, D. M. Zajac, P. W. Deelman, and J. R. Petta, Strong coupling of a single electron in silicon to a microwave photon, Science 355, 156 (2017).

[26] X. Mi, M. Benito, S. Putz, D. M. Zajac, J. M. Taylor, G. Burkard, and J. R. Petta, A coherent spin-photon interface in silicon, Nature 555, 599 (2018).

[27] A. J. Landig, J. V. Koski, P. Scarlino, U. C. Mendes, A. Blais, C. Reichl, W. Wegscheider, A. Wallraff, K. Ensslin, and T. Ihn, Coherent spin-photon coupling using a resonant exchange qubit, Nature 560, 179 (2018).

[28] R. Vyas and S. Singh, Photon-counting statistics of the degenerate optical parametric oscillator, Phys. Rev. A 40, 5147 (1989).

[29] H. J. Carmichael, S. Singh, R. Vyas, and P. R. Rice, Photoelectron waiting times and atomic state reduction in resonance fluorescence, Phys. Rev. A 39, 1200 (1989).

[30] X. H. H. Zhang and H. U. Baranger, Quantum interference and complex photon statistics in waveguide QED, Phys. Rev. A 97, 023813 (2018).

[31] M. Albert, G. Haack, C. Flindt, and M. Büttiker, Electron Waiting Times in Mesoscopic Conductors, Phys. Rev. Lett. 108, 186806 (2012).

[32] G. Haack, M. Albert, and C. Flindt, Distributions of electron waiting times in quantum-coherent conductors, Phys. Rev. B 90, 205429 (2014).

[33] D. Dasenbrook, P. P. Hofer, and C. Flindt, Electron waiting times in coherent conductors are correlated, Phys. Rev. B 91, 195420 (2015).

[34] C. W. J. Beenakker, Thermal Radiation and Amplified Spontaneous Emission from a Random Medium, Phys. Rev. Lett. 81, 1829 (1998).

[35] C. W. J. Beenakker and H. Schomerus, Counting Statistics of Photons Produced by electronic Shot Noise, Phys. Rev. Lett. 86, 700 (2001).

[36] D. Kambly, C. Flindt, and M. Büttiker, Factorial cumulants reveal interactions in counting statistics, Phys. Rev. B 83, 075432 (2011).

[37] D. Kambly and C. Flindt, Time-dependent factorial cumulants in interacting nano-scale systems, J. Comp. Electron. 12, 331 (2013).

[38] P. Stegmann, B. Sothmann, A. Hucht, and J. König, Detection of interactions via generalized factorial cumulants in 
systems in and out of equilibrium, Phys. Rev. B 92, 155413 (2015).

[39] E. Kleinherbers, P. Stegmann, and J. König, Revealing attractive electron-electron interaction in a quantum dot by full counting statistics, New J. Phys. 20, 073023 (2018).

[40] Y. Komijani, T. Choi, F. Nichele, K. Ensslin, T. Ihn, D. Reuter, and A. D. Wieck, Counting statistics of hole transfer in a $p$-type gaas quantum dot with dense excitation spectrum, Phys. Rev. B 88, 035417 (2013).

[41] K. Saito and A. Dhar, Fluctuation Theorem in Quantum Heat Conduction, Phys. Rev. Lett. 99, 180601 (2007).

[42] J. R. Gomez-Solano, L. Bellon, A. Petrosyan, and S. Ciliberto, Steady-state fluctuation relations for systems driven by an external random force, Europhys. Lett. 89, 60003 (2010).

[43] H. P. Breuer and F. Petruccione, The Theory of Open Quantum Systems (Oxford University Press, Oxford, 2007).

[44] M. B. Plenio and P. L. Knight, The quantum-jump approach to dissipative dynamics in quantum optics, Rev. Mod. Phys. 70, 101 (1998).

[45] M. Renardy and R. C. Rogers, An Introduction to Partial Differential Equations, 2nd ed. (Springer, New York, 2004).

[46] H. F. Arnoldus, Density matrix for photons in a cavity, J. Opt. Soc. Am. B 13, 1099 (1996).

[47] A. A. Clerk and D. W. Utami, Using a qubit to measure photonnumber statistics of a driven thermal oscillator, Phys. Rev. A 75, 042302 (2007).

[48] A. A. Clerk, Full counting statistics of energy fluctuations in a driven quantum resonator, Phys. Rev. A 84, 043824 (2011).

[49] P. P. Hofer and A. A. Clerk, Negative Full Counting Statistics Arise from Interference Effects, Phys. Rev. Lett. 116, 013603 (2016).

[50] G. Bédard, Photon counting statistics of Gaussian light, Phys. Rev. 151, 1038 (1966).

[51] N. Tornau and B. Echtermeyer, Photon counting statistics of thermal light consisting of two spectral lines, Ann. Phys. 484, 289 (1973).

[52] C. L. Mehta and S. Gupta, Photon counting statistics with thermal light having a multiple-peak spectrum, Phys. Rev. A 11, 1634 (1975).

[53] The Lindblad equation is valid for $\tau_{B}, 1 / \omega_{0} \ll 1 / \gamma$, where $\tau_{B} \sim \hbar /\left(k_{B} T\right)$ is the bath correlation time. The coarse-grained description of bath excitations, which are assumed to decay much faster than the system evolves, implies that our results are valid for times that are longer than the bath correlation time, $t \gg \tau_{B}$, or on frequencies, $\omega \ll 1 / \tau_{B} \sim k_{B} T / \hbar$, that are smaller than the temperature [43]. Realistic parameters are $\omega_{0} \simeq 5 \mathrm{GHz}, \gamma \simeq 50 \mathrm{MHz}$, and $\tau_{B} \simeq 0.1 \mathrm{~ns}$ at $T=100 \mathrm{mK}$, showing that these assumptions are experimentally justified.

[54] S. K. Gorman, Y. He, M. G. House, J. G. Keizer, D. Keith, L. Fricke, S. J. Hile, M. A. Broome, and M. Y. Simmons, Tunneling Statistics for Analysis of Spin-Readout Fidelity, Phys. Rev. Appl. 8, 034019 (2017).

[55] A. Öttl, S. Ritter, M. Köhl, and T. Esslinger, Correlations and Counting Statistics of an Atom Laser, Phys. Rev. Lett. 95, 090404 (2005).

[56] C. Emary, C. Pöltl, A. Carmele, J. Kabuss, A. Knorr, and T. Brandes, Bunching and antibunching in electronic transport, Phys. Rev. B 85, 165417 (2012).
[57] F. Hassler, M. V. Suslov, G. M. Graf, M. V. Lebedev, G. B. Lesovik, and G. Blatter, Wave-packet formalism of full counting statistics, Phys. Rev. B 78, 165330 (2008).

[58] A. G. Abanov and D. A. Ivanov, Allowed Charge Transfers Between Coherent Conductors Driven by a Time-Dependent Scatterer, Phys. Rev. Lett. 100, 086602 (2008).

[59] A. G. Abanov and D. A. Ivanov, Factorization of quantum charge transport for noninteracting fermions, Phys. Rev. B 79, 205315 (2009).

[60] H. Touchette, The large deviation approach to statistical mechanics, Phys. Rep. 478, 1 (2009).

[61] C. Bergenfeldt, P. Samuelsson, B. Sothmann, C. Flindt, and M. Büttiker, Hybrid Microwave-Cavity Heat Engine, Phys. Rev. Lett. 112, 076803 (2014).

[62] G. Tang and J.-S. Wang, Heat transfer statistics in extreme-nearfield radiation, Phys. Rev. B 98, 125401 (2018).

[63] T. Denzler and E. Lutz, Heat distribution of a quantum harmonic oscillator, Phys. Rev. E 98, 052106 (2018).

[64] D. S. P. Salazar, A. M. S. Macêdo, and G. L. Vasconcelos, Heat distribution in open quantum systems with maximum entropy production, arXiv:1811.07870.

[65] The moment-generating function of the net transfer of photons reduces to the expression in Eq. (3) if one reservoir is much colder than the other, see Appendix F.

[66] D. S. Golubev and J. P. Pekola, Statistics of heat exchange between two resistors, Phys. Rev. B 92, 085412 (2015).

[67] G. Gallavotti and E. G. D. Cohen, Dynamical ensembles in stationary states, J. Stat. Phys. 80, 931 (1995).

[68] G. Gallavotti and E. G. D. Cohen, Dynamical Ensembles in Nonequilibrium Statistical Mechanics, Phys. Rev. Lett. 74, 2694 (1995).

[69] R. Sánchez, R. López, D. Sánchez, and M. Büttiker, Mesoscopic Coulomb Drag, Broken Detailed Balance, and Fluctuation Relations, Phys. Rev. Lett. 104, 076801 (2010).

[70] D. K. C. Macdonald, Spontaneous fluctuations, Rep. Prog. Phys. 12, 56 (1949).

[71] C. Flindt, T. Novotný, and A.-P. Jauho, Current noise spectrum of a quantum shuttle, Physica E 29, 411 (2005).

[72] N. Lambert, R. Aguado, and T. Brandes, Nonequilibrium entanglement and noise in coupled qubits, Phys. Rev. B 75, 045340 (2007).

[73] D. V. Averin and J. P. Pekola, Violation of the FluctuationDissipation Theorem in Time-Dependent Mesoscopic Heat Transport, Phys. Rev. Lett. 104, 220601 (2010).

[74] R. Kubo, The fluctuation-dissipation theorem, Rep. Prog. Phys. 29, 255 (1966).

[75] G. Lindblad, On the generators of quantum dynamical semigroups, Commun. Math. Phys. 48, 119 (1976).

[76] V. Gorini, A. Kossakowski and E. C. G. Sudarshan, Completely positive dynamical semigroups of N-level systems, J. Math. Phys. 17, 821 (1976).

[77] D. A. Bagrets and Yu. V. Nazarov, Full counting statistics of charge transfer in Coulomb blockade systems, Phys. Rev. B 67, 085316 (2003).

[78] L. S. Levitov, H. Lee and G. B. Lesovik, Electron counting statistics and coherent states of electric current, J. Math. Phys. 37, 4845 (1996).

[79] C. Flindt, T. Novotný and A.-P. Jauho, Full counting statistics of nano-electromechanical systems, Europhys. Lett. 69, 475 (2004). 